\title{
Jacques Tits motivic measure
}

\author{
Gonçalo Tabuada ${ }^{1,2,3}$ (I)
}

Received: 18 January 2021 / Revised: 7 September 2021 / Accepted: 8 October 2021 /

Published online: 6 November 2021

(C) The Author(s) 2021

\begin{abstract}
In this article we construct a new motivic measure called the Jacques Tits motivic measure. As a first main application, we prove that two Severi-Brauer varieties (or, more generally, two twisted Grassmannian varieties), associated to 2-torsion central simple algebras, have the same class in the Grothendieck ring of varieties if and only if they are isomorphic. In addition, we prove that if two Severi-Brauer varieties, associated to central simple algebras of period $\{3,4,5,6\}$, have the same class in the Grothendieck ring of varieties, then they are necessarily birational to each other. As a second main application, we prove that two quadric hypersurfaces (or, more generally, two involution varieties), associated to quadratic forms of dimension 6 or to quadratic forms of arbitrary dimension defined over a base field $k$ with $I^{3}(k)=0$, have the same class in the Grothendieck ring of varieties if and only if they are isomorphic. In addition, we prove that the latter main application also holds for products of quadric hypersurfaces.
\end{abstract}

\section{Introduction}

Let $k$ be a field and $\operatorname{Var}(k)$ the category of varieties, i.e., reduced separated $k$-schemes of finite type. The Grothendieck ring of varieties $K_{0} \operatorname{Var}(k)$, introduced in a letter from

Communicated by Vasudevan Srinivas.

The author was partially supported by the Huawei-IHÉS research funds and by the FCT - Fundação para a Ciência e a Tecnologia, I.P., under the scope of the project UIDB/00297/2020 (Center for Mathematics and Applications).

$凶$ Gonçalo Tabuada goncalo.tabuada@warwick.ac.uk https://homepages.warwick.ac.uk/ u1972846/

1 Mathematics Institute, University of Warwick, Zeeman Building, Coventry CV4 7AL, UK

2 Departamento de Matemática, FCT, UNL, Lisbon, Portugal

3 Centro de Matemática e Aplicações (CMA), FCT, UNL, Lisbon, Portugal 
Grothendieck to Serre (consult [7, letter of 16/08/1964]), is defined as the quotient of the free abelian group on the set of isomorphism classes of varieties $[X]$ by the "scissor" relations $[X]=[Y]+[X \backslash Y]$, where $Y$ is a closed subvariety of $X$. The multiplication law is induced by the product of varieties. Despite the efforts of several mathematicians (consult, for example, the works of Bittner [3] and Larsen-Lunts [19]), the structure of the Grothendieck ring of varieties still remains nowadays poorly understood. In order to capture some of its flavor, a few motivic measures, i.e., ring homomorphisms $\mu: K_{0} \operatorname{Var}(k) \rightarrow R$, have been built. For example, when $k$ is finite the assignment $X \mapsto \# X(k)$ gives rise to the counting motivic measure $\mu_{\#}: K_{0} \operatorname{Var}(k) \rightarrow \mathbb{Z}$, and when $k=\mathbb{C}$ the assignment $X \mapsto \chi(X):=\Sigma_{n}(-1)^{n} \operatorname{dim}_{\mathbb{Q}} H_{c}^{n}\left(X^{\mathrm{an}} ; \mathbb{Q}\right)$ gives rise to the Euler characteristic motivic measure $\mu_{\chi}: K_{0} \operatorname{Var}(k) \rightarrow \mathbb{Z}$. In this article we construct a new motivic measure $\mu_{\mathrm{JT}}$ called the Jacques Tits motivic measure. Making use of it, we then establish several new properties of the Grothendieck ring of varieties.

\section{Statement of results}

Let $k$ be a field of characteristic zero and $\Gamma:=\operatorname{Gal}(\bar{k} / k)$ its absolute Galois group.

Recall that given a split semi-simple algebraic group $G$ over $k$, a parabolic subgroup $P \subset G$, and a 1-cocycle $\gamma: \Gamma \rightarrow G(\bar{k})$, we can consider the projective homogeneous variety $\mathcal{F}:=G / P$ as well as its twisted form ${ }_{\gamma} \mathcal{F}$. Let us write $\widetilde{G}$ and $\widetilde{P}$ for the universal covers of $G$ and $P$, respectively, $R(\widetilde{G})$ and $R(\widetilde{P})$ for the associated representation rings, $n(\mathcal{F})$ for the index $[W(\widetilde{G}): W(\widetilde{P})]$ of the Weyl groups, $\widetilde{Z}$ for the center of $\widetilde{G}$, and $C h$ for the character group $\operatorname{Hom}\left(\widetilde{Z}, \mathbb{G}_{m}\right)$. As proved by Steinberg in [24] (consult also [22, $\S 12.5-\S 12.8]$, we have $R(\widetilde{P})=\bigoplus_{i} R(\widetilde{G}) \rho_{i}$, where $\left\{\rho_{i}\right\}_{i}$ is a canonical $C h$ homogeneous basis of cardinality $n(\mathcal{F})$. Let us denote by $A_{\rho_{i}}$ the Tits central simple $k$-algebra associated to $\rho_{i}$; consult $[12, \S 27]$ [30] for details.

Notation 1.1 Let us write $K_{0} \operatorname{Var}(k)^{\mathrm{tw}}$ for the smallest subring of $K_{0} \operatorname{Var}(k)$ containing the Grothendieck classes $\left[{ }_{\gamma} \mathcal{F}\right]$ of all twisted projective homogeneous varieties ${ }_{\gamma} \mathcal{F}$.

Consider the Brauer group $\operatorname{Br}(k)$ of $k$, the associated group ring $\mathbb{Z}[\operatorname{Br}(k)]$, and the following quotient ring

$$
R_{\mathrm{B}}(k):=\mathbb{Z}[\operatorname{Br}(k)] /\left\langle[k]+\left[A \otimes A^{\prime}\right]-[A]-\left[A^{\prime}\right] \mid\left(\operatorname{ind}(A), \operatorname{ind}\left(A^{\prime}\right)\right)=1\right\rangle,
$$

where $A$ and $A^{\prime}$ are central simple $k$-algebras with coprime indexes. Note that in the particular case where there exists a prime number $q$ such that every element of $\operatorname{Br}(k)$ is of $q$-primary torsion, the quotient ring $R_{\mathrm{B}}(k)$ agrees with the group ring $\mathbb{Z}[\operatorname{Br}(k)]$.

Our main result is the following:

Theorem 1.2 The assignment $\gamma \mathcal{F} \mapsto \Sigma_{i}\left[A_{\rho_{i}}\right]$ gives rise to a motivic measure $\mu_{\mathrm{JT}}: K_{0} \operatorname{Var}(k)^{\mathrm{tw}} \rightarrow R_{\mathrm{B}}(k)$.

Intuitively speaking, Theorem 1.2 shows that the Tits algebras associated to a twisted projective homogeneous variety are preserved by the "scissor" relations. Motivated by this fact, we decided to call $\mu_{\mathrm{JT}}$ the Jacques Tits motivic measure or, more simply, the Tits motivic measure. The proof of Theorem 1.2 makes use, among other ingredients, of the recent theory of noncommutative motives; consult $\$ 3-\$ 4$ below. 


\section{Applications}

In this section we describe several applications of the Tits motivic measure to Severi-Brauer varieties, twisted Grassmannian varieties, quadric hypersurfaces, and involution varieties.

Notation 2.1 Given a central simple $k$-algebra $A$, let us write $\operatorname{deg}(A)$ for its degree, ind $(A)$ for its index, $\operatorname{per}(A)$ for its period, $[A]$ for its class in the Brauer group $\operatorname{Br}(k)$, and finally $\langle[A]\rangle$ for the subgroup of $\operatorname{Br}(k)$ generated by $[A]$.

\subsection{Severi-Brauer varieties}

Let $G$ be the projective general linear group $P G L_{n}$, with $n \geq 2$. In this case, we have $\widetilde{G}=S L_{n}$. Consider the following parabolic subgroup:

$$
\widetilde{P}:=\left\{\left(\begin{array}{ll}
a & b \\
0 & c
\end{array}\right) \mid a \cdot \operatorname{det}(c)=1\right\} \subset S L_{n} a \in k^{\times} c \in G L_{n-1} .
$$

The associated projective homogeneous variety $\mathcal{F}:=G / P \simeq \widetilde{G} / \widetilde{P}$ is the projective space $\mathbb{P}^{n-1}$ and we have $R(\widetilde{P})=\oplus_{i=0}^{n-1} R(\widetilde{G}) \rho_{i}$. Given a 1-cocycle $\gamma: \Gamma \rightarrow$ $P G L_{n}(\bar{k})$, let $A$ be the corresponding central simple $k$-algebra of degree $n$. Under these notations, the twisted form $\gamma \mathbb{P}^{n-1}$ is the Severi-Brauer variety $\operatorname{SB}(A)$ and the Tits central simple $k$-algebra $A_{\rho_{i}}$ is the tensor product $A^{\otimes i}$.

Theorem 2.2 Let $A$ and $A^{\prime}$ be two central simple $k$-algebras. If $[\mathrm{SB}(A)]=\left[\operatorname{SB}\left(A^{\prime}\right)\right]$ in the Grothendieck ring of varieties $K_{0} \operatorname{Var}(k)$, then the following holds:

(i) We have $\operatorname{dim}(\operatorname{SB}(A))=\operatorname{dim}\left(\operatorname{SB}\left(A^{\prime}\right)\right)$. Equivalently, we have $\operatorname{deg}(A)=\operatorname{deg}\left(A^{\prime}\right)$.

(ii) We have $\langle[A]\rangle=\left\langle\left[A^{\prime}\right]\right\rangle$. In particular, we have $\operatorname{per}(A)=\operatorname{per}\left(A^{\prime}\right)$.

(iii) When $[A] \in{ }_{2} \operatorname{Br}(k)$, where ${ }_{2} \operatorname{Br}(k)$ is the 2-torsion subgroup of $\operatorname{Br}(k)$, we have $\mathrm{SB}(A) \simeq \mathrm{SB}\left(A^{\prime}\right)$.

(iv) When $\operatorname{per}(A) \in\{3,4,5,6\}$, the Severi-Brauer varieties $\mathrm{SB}(A)$ and $\mathrm{SB}\left(A^{\prime}\right)$ are birational to each other.

(v) The Severi-Brauer varieties $\mathrm{SB}(A)$ and $\mathrm{SB}\left(A^{\prime}\right)$ are stably birational to each other.

Note that item (i), resp. item (ii), shows that the dimension of a Severi-Brauer variety, resp. the subgroup generated by the Brauer class, is preserved by the "scissor" relations. Item (iii) shows that when the Brauer class is 2-torsion (i.e., when $\operatorname{per}(A) \in\{1,2\}$ ), two Severi-Brauer varieties have the same Grothendieck class in $K_{0} \operatorname{Var}(k)$ if and only if they are isomorphic! In other words, item (iii) yields the following inclusion:

$$
\begin{aligned}
& \frac{\left\{\text { Severi-Brauer varieties } \operatorname{SB}(A) \text { with }[A] \in{ }_{2} \operatorname{Br}(k)\right\}}{\text { isomorphism }} \\
& \subset K_{0} \operatorname{Var}(k) .
\end{aligned}
$$

Note that thanks to the Artin-Wedderburn theorem, the left-hand side of (2.3) is in bijection with ${ }_{2} \operatorname{Br}(k) \times \mathbb{N}$ via the assignment $\mathrm{SB}(A) \mapsto\left([A], \frac{\operatorname{deg}(A)}{\operatorname{ind}(A)}\right)$. Note also 
that by restricting the inclusion (2.3) to central simple $k$-algebras of degree 2 , i.e., to quaternion algebras $Q=(a, b)$, we obtain the following inclusion

$$
\frac{\left\{C(a, b):=\left(a x^{2}+b y^{2}-z^{2}=0\right) \subset \mathbb{P}^{2} \mid a, b \in k^{\times}\right\}}{\text {isomorphism }} \subset K_{0} \operatorname{Var}(k),
$$

where $C(a, b)$ stands for the smooth conic associated to the quaternion algebra $Q$.

Example 2.5 (Conics over $\mathbb{Q}$ ) When $k=\mathbb{Q}$, there are infinitely many smooth conics in $\mathbb{P}^{2}$ up to isomorphism. For example, given any two primes numbers $p \neq q$ which are congruent to 3 modulo 4 , the conics $C(-1, p)$ and $C(-1, q)$ are not isomorphic. Consequently, since there are infinitely many prime numbers $p$ which are congruent to 3 modulo 4, the inclusion (2.4) yields the following infinite family of distinct Grothendieck classes $\left\{\left[\left(-x^{2}+p y^{2}-z^{2}=0\right)\right]\right\}_{p \equiv 3(\bmod 4)} \subset K_{0} \operatorname{Var}(\mathbb{Q})$.

Item (iv) shows that, for small values of the period, if two Severi-Brauer varieties have the same Grothendieck class in $K_{0} \operatorname{Var}(k)$ then they are necessarily birational to each other.

Remark 2.6 (Severi-Brauer surfaces) Let $A$ and $A^{\prime}$ be two central simple $k$-algebras of degree 3 (and hence of period 3). In this particular case, Hogadi proved in [10, Thm. 1.2], using different arguments, that if $[\mathrm{SB}(A)]=\left[\mathrm{SB}\left(A^{\prime}\right)\right]$ in the Grothendieck ring of varieties $K_{0} \operatorname{Var}(k)$, then the Severi-Brauer varieties $\mathrm{SB}(A)$ and $\mathrm{SB}\left(A^{\prime}\right)$ are birational to each other. Note that, in contrast with Hogadi, in item (iv) we do not impose any restriction on the degree (only on the period).

Remark 2.7 (Amitsur conjecture) Let $A$ and $A^{\prime}$ be two central simple $k$-algebras with the same degree. In the fifties, Amitsur [2] conjectured that if $\langle[A]\rangle=\left\langle\left[A^{\prime}\right]\right\rangle$, then the Severi-Brauer varieties $\mathrm{SB}(A)$ and $\mathrm{SB}\left(A^{\prime}\right)$ are birational to each other. This conjecture holds for example, when ind $(A)<\operatorname{deg}(A)$ (for every $A^{\prime}$ ) or when $k$ is a local or global field (for every $A$ and $A^{\prime}$ ). Therefore, items (i)-(ii) of Theorem 2.2 yield the following (conditional) result:

Corollary 2.8 Let $A$ and $A^{\prime}$ be two central simple $k$-algebras for which the Amitsur conjecture holds. If $[\mathrm{SB}(A)]=\left[\mathrm{SB}\left(A^{\prime}\right)\right]$ in the Grothendieck ring of varieties $K_{0} \operatorname{Var}(k)$, then the Severi-Brauer varieties $\mathrm{SB}(A)$ and $\mathrm{SB}\left(A^{\prime}\right)$ are birational to each other.

Finally, item (v) shows that if two Severi-Brauer varieties have the same Grothendieck class in $K_{0} \operatorname{Var}(k)$, then they are necessarily stably birational to each other. As proved by Larsen-Lunts in [19, Cor. 2.6], this result holds in much greater generality: given two smooth proper varieties $X$ and $Y$, if $[X]=[Y]$ in the Grothendieck ring of varieties $K_{0} \operatorname{Var}(k)$, then $X$ and $Y$ are stably birational to each other.

\subsection{Products of conics}

Recall that two quaternion $k$-algebras $Q$ and $Q^{\prime}$ are called unlinked in the sense of Albert [1] if their tensor product $Q \otimes Q^{\prime}$ is a division $k$-algebra. 
Proposition 2.9 Let $Q=(a, b), Q^{\prime}=\left(a^{\prime}, b^{\prime}\right), Q^{\prime \prime}=\left(a^{\prime \prime}, b^{\prime \prime}\right), Q^{\prime \prime \prime}=\left(a^{\prime \prime \prime}, b^{\prime \prime \prime}\right)$ be four quaternion algebras. If $\left[C(a, b) \times C\left(a^{\prime}, b^{\prime}\right)\right]=\left[C\left(a^{\prime \prime}, b^{\prime \prime}\right) \times C\left(a^{\prime \prime \prime}, b^{\prime \prime \prime}\right)\right]$ in $K_{0} \operatorname{Var}(k)$, then the following holds:

(i) The conic $C(a, b)\left(\right.$ or $\left.C\left(a^{\prime}, b^{\prime}\right)\right)$ is isomorphic to $C\left(a^{\prime \prime}, b^{\prime \prime}\right)$ or to $C\left(a^{\prime \prime \prime}, b^{\prime \prime \prime}\right)$.

(ii) When $Q$ and $Q^{\prime}$ are unlinked, we have $C(a, b) \times C\left(a^{\prime}, b^{\prime}\right) \simeq C\left(a^{\prime \prime}, b^{\prime \prime}\right) \times$ $C\left(a^{\prime \prime \prime}, b^{\prime \prime \prime}\right)$.

Note that item (i) shows that if two products of conics have the same Grothendieck class in $K_{0} \operatorname{Var}(k)$, then they necessarily share a common conic! Moreover, item (ii) provides a sufficient condition for these two products of conics to be isomorphic.

Example 2.10 (Unlinked quaternion algebras) When $k=\mathbb{R}(x, y)$ is the field of rational functions on two variables over $\mathbb{R}$, the quaternion algebras $(-1,-1)$ and $(x, y)$, as well as the quaternion algebras $(x,-1)$ and $(-x, y)$, are unlinked; consult $[18, \S \mathrm{VI}$ Examples 1.11 and 1.13]. In the same vein, when $k=\mathbb{Q}(x, y)$ is the field of rational functions on two variables over $\mathbb{Q}$, the quaternion algebras $(a, x)$ and $(b, y)$, where $a, b \in k^{\times}$represent two independent square classes in $\mathbb{Q}^{\times} /\left(\mathbb{Q}^{\times}\right)^{2}$, are unlinked; consult [18, §VI Example 1.15]. Further examples exist for every field $k$ with $u$ invariant equal to 6 or $>8$; consult $[18, \S \mathrm{XIII}]$.

Remark 2.11 (Birationality) Let $k$ be a number field or the function field of an algebraic surface over $\mathbb{C}$. Given quaternion algebras $Q=(a, b), Q^{\prime}=\left(a^{\prime}, b^{\prime}\right), Q^{\prime \prime}=\left(a^{\prime \prime}, b^{\prime \prime}\right)$, and $Q^{\prime \prime \prime}=\left(a^{\prime \prime \prime}, b^{\prime \prime \prime}\right)$, Kollár $^{1}$ proved in [13, Thm. 2] that if $\left[C(a, b) \times C\left(a^{\prime}, b^{\prime}\right)\right]=$ $\left[C\left(a^{\prime \prime}, b^{\prime \prime}\right) \times C\left(a^{\prime \prime \prime}, b^{\prime \prime \prime}\right)\right]$ in the Grothendieck group of varieties $K_{0} \operatorname{Var}(k)$, then the products $C(a, b) \times C\left(a^{\prime}, b^{\prime}\right)$ and $C\left(a^{\prime \prime}, b^{\prime \prime}\right) \times C\left(a^{\prime \prime \prime}, b^{\prime \prime \prime}\right)$ are birational to each other. Hence, the above Proposition 2.9 may be understood as a refinement of Kollar's result.

\subsection{Twisted Grassmannian varieties}

Let $G=P G L_{n}$, with $n \geq 2$. Recall that in this case we have $\widetilde{G}=S L_{n}$. Choose an integer $1 \leq d<n$ and consider the following parabolic subgroup:

$$
\widetilde{P}:=\left\{\left(\begin{array}{ll}
a & b \\
0 & c
\end{array}\right) \mid \operatorname{det}(a) \cdot \operatorname{det}(c)=1\right\} \subset S L_{n} a \in G L_{d} c \in G L_{n-d} \text {. }
$$

The associated projective homogeneous variety $\mathcal{F}:=G / P \simeq \widetilde{G} / \widetilde{P}$ is the Grassmannian variety $\operatorname{Gr}(d)$ and we have $R(\widetilde{P})=\oplus_{i} R(\widetilde{G}) \rho_{i}$ where $i=\left(i_{1}, \ldots, i_{d}\right)$ is a Young diagram inside the rectangle with $d$ lines and $n-d$ columns. Given a 1-cocycle $\gamma: \Gamma \rightarrow P G L_{n}(\bar{k})$, let $A$ be the corresponding central simple $k$-algebra of degree $n$. Under these notations, the twisted form ${ }_{\gamma} \operatorname{Gr}(d)$ is the twisted Grassmannian variety $\operatorname{Gr}(d ; A)$ and the Tits central simple $k$-algebra $A_{\rho_{i}}$ is the tensor product $A^{\otimes\left(i_{1}+\cdots+i_{d}\right)}$.

Remark 2.12 (Generalization) Note that in the particular case where $d=1$, the twisted Grassmannian variety $\operatorname{Gr}(d ; A)$ reduces to the Severi-Brauer variety $\operatorname{SB}(A)$.

\footnotetext{
1 In subsequent work, Hogadi [10] removed these restrictions on the base field $k$.
} 
Theorem 2.13 Let $A$ and $A^{\prime}$ be two central simple $k$-algebras and $1 \leq d<\operatorname{deg}(A)$ and $1 \leq d^{\prime}<\operatorname{deg}\left(A^{\prime}\right)$. If $[\operatorname{Gr}(d ; A)]=\left[\operatorname{Gr}\left(d^{\prime} ; A^{\prime}\right)\right]$ in the Grothendieck ring of varieties $K_{0} \operatorname{Var}(k)$, then the following holds:

(i) We have $\operatorname{dim}(\operatorname{Gr}(d ; A))=\operatorname{dim}\left(\operatorname{Gr}\left(d^{\prime} ; A^{\prime}\right)\right)$. Moreover, we have $\operatorname{deg}(A)=$ $\operatorname{deg}\left(A^{\prime}\right)$.

(ii) We have $\langle[A]\rangle=\left\langle\left[A^{\prime}\right]\right\rangle$. In particular, we have $\operatorname{per}(A)=\operatorname{per}\left(A^{\prime}\right)$.

(iii) When $[A] \in{ }_{2} \operatorname{Br}(k)$, we have $\operatorname{Gr}(d ; A) \simeq \operatorname{Gr}\left(d^{\prime} ; A^{\prime}\right)$.

Note that, similarly to Theorem 2.2, item (iii) shows that when the Brauer class is 2-torsion, two twisted Grassmannian varieties have the same Grothendieck class in $K_{0} \operatorname{Var}(k)$ if and only if they are isomorphic! In other words, item (iii) yields the following inclusion:

$\left\{\right.$ Twisted Grassmannian varieties $\operatorname{Gr}(d ; A)$ with $\left.[A] \in{ }_{2} \operatorname{Br}(k)\right\}$ isomorphism $\subset K_{0} \operatorname{Var}(k)$.

Following Remark 2.12, note that (2.14) extends the above inclusion (2.3).

\subsection{Quadric hypersurfaces}

Let $G$ be the special orthogonal group $S O_{n}$, with $n \geq 3$, with respect to the hyperbolic form $\frac{n}{2} \mathbb{H}$ when $n$ is even or to the form $\left\lfloor\frac{n}{2}\right\rfloor \mathbb{H} \perp\langle 1\rangle$ when $n$ is odd. In this case, we have $\widetilde{G}=\operatorname{Spin}_{n}$. Consider the action of $G$ on $\mathbb{P}^{n-1}$ given by projective linear transformations, the stabilizer $P \subset G$ of the isotropic point $[1: 0: \cdots: 0]$, and the pre-image $\widetilde{P} \subset \widetilde{G}$ of $P$. The associated projective homogeneous variety $\mathcal{F}:=$ $G / P \simeq \widetilde{G} / \widetilde{P}$ is the following smooth quadric hypersurface

$$
Q:= \begin{cases}\left(x_{1} y_{1}+\cdots+x_{\frac{n}{2}} y_{\frac{n}{2}}=0\right) \subset \mathbb{P}^{n-1} & n \text { even } \\ \left(x_{1} y_{1}+\cdots+x_{\left\lfloor\frac{n}{2}\right\rfloor} y_{\left\lfloor\frac{n}{2}\right\rfloor}+z^{2}=0\right) \subset \mathbb{P}^{n-1} & n \text { odd }\end{cases}
$$

and we have $R(\widetilde{P})=\oplus_{i=0}^{n-1} R(\widetilde{G}) \rho_{i}$ when $n$ is even or $R(\widetilde{P})=\oplus_{i=0}^{n-2} R(\widetilde{G}) \rho_{i}$ when $n$ is odd. Given a 1-cocycle $\gamma: \Gamma \rightarrow S O_{n}(\bar{k})$, let $q$ be the corresponding non-degenerate quadratic form with trivial discriminant of dimension $n$. Under these notations, the twisted form ${ }_{\gamma} Q$ is the smooth quadric hypersurface $Q_{q} \subset \mathbb{P}^{n-1}$ and the Tits central simple $k$-algebra $A_{\rho_{i}}$ is given as follows

$$
A_{\rho_{i}}:=\left\{\begin{array}{ll}
k & 0 \leq i \leq n-3 \quad n \text { even } \\
C_{0}^{+}(q) & i=n-2 \quad n \text { even } \\
C_{0}^{-}(q) & i=n-1 \quad n \text { even }
\end{array} \quad A_{\rho_{i}}:= \begin{cases}k & 0 \leq i \leq n-3 n \text { odd } \\
C_{0}(q) & i=n-2 n \text { odd },\end{cases}\right.
$$

where $C_{0}(q)$ stands for the even Clifford algebra of $q$ and $C_{0}^{+}(q)$ and $C_{0}^{-}(q)$ for the (isomorphic) simple components of $C_{0}(q)$. 
Theorem 2.15 Let $q$ and $q^{\prime}$ be two non-degenerate quadratic forms with trivial discriminant of dimensions $n$ and $n^{\prime}$, respectively. If $\left[Q_{q}\right]=\left[Q_{q^{\prime}}\right]$ in the Grothendieck ring of varieties $K_{0} \operatorname{Var}(k)$, then the following holds:

(i) We have $\operatorname{dim}\left(Q_{q}\right)=\operatorname{dim}\left(Q_{q^{\prime}}\right)$. Equivalently, we have $n=n^{\prime}$.

(ii) We have $C_{0}^{+}(q) \simeq C_{0}^{+}\left(q^{\prime}\right)$ when $n$ is even or $C_{0}(q) \simeq C_{0}\left(q^{\prime}\right)$ when $n$ is odd.

(iii) When $n=6$, we have $Q_{q} \simeq Q_{q^{\prime}}$.

(iv) When $I^{3}(k)=0$, where $I(k) \subset W(k)$ is the fundamental ideal of the Witt ring, we have $Q_{q} \simeq Q_{q^{\prime}}$.

Note that item (i), resp. item (ii), shows that the dimension of the quadric hypersurface, resp. the Brauer class of the (simple components of the) even Clifford algebra, is preserved by the "scissor" relations. Item (iii) shows that when the dimension is equal to 6, two quadric hypersurfaces have the same Grothendieck class in $K_{0} \operatorname{Var}(k)$ if and only if they are isomorphic! Recall from [12, §16.4] that, up to similarity, a non-degenerate quadratic form $q$ with trivial discriminant of dimension 6 is given by $\left\langle a, b,-a b,-a^{\prime},-b^{\prime}, a^{\prime} b^{\prime}\right\rangle$ with $a, b, a^{\prime}, b^{\prime} \in k^{\times}$. Therefore, item (iii) yields the following inclusion:

$$
\frac{\left\{Q_{q}:=\left(a u^{2}+b v^{2}-a b w^{2}-a^{\prime} x^{2}-b^{\prime} y^{2}+a^{\prime} b^{\prime} z^{2}=0\right) \subset \mathbb{P}^{5} \mid a, b, a^{\prime}, b^{\prime} \in k^{\times}\right\}}{\text {isomorphism }} \subset K_{0} \operatorname{Var}(k) .
$$

Example 2.17 (Quadric hypersurfaces over $\mathbb{Q}$ ) When $k=\mathbb{Q}$, there are infinitely many quadric hypersurfaces in $\mathbb{P}^{5}$ up to isomorphism. For example, we have the following infinite family of non-isomorphic quadric hypersurfaces $\left\{\left(u^{2}+v^{2}-w^{2}+x^{2}-p y^{2}-\right.\right.$ $\left.\left.p z^{2}=0\right)\right\}_{p \equiv 3(\bmod 4)}$ parametrized by the prime numbers $p$ which are congruent to 3 modulo 4. Making use of (2.16), we hence obtain the following infinite family of distinct Grothendieck classes $\left\{\left[\left(u^{2}+v^{2}-w^{2}+x^{2}-p y^{2}-p z^{2}=0\right)\right]\right\}_{p \equiv 3(\bmod 4)} \subset$ $K_{0} \operatorname{Var}(\mathbb{Q})$. Note that since all these quadric hypersurfaces have a rational $k$-point, it follows from [14, Thm. 1.11] that they are all birational to $\mathbb{P}^{4}$. This shows that, in the case of quadric hypersurfaces, the Grothendieck class in $K_{0} \operatorname{Var}(k)$ contains much more information than the birational equivalence class.

Finally, item (iv) shows that when $I^{3}(k)=0$, two quadric hypersurfaces have the same Grothendieck class in $K_{0} \operatorname{Var}(k)$ if and only if they are isomorphic! Consequently, in this case, the above inclusion (2.16) admits the following far-reaching extension:

$$
\frac{\text { QQuadric hypersurfaces } \left.Q_{q} \text { with trivial discriminant }\right\}}{\text { isomorphism }} \subset K_{0} \operatorname{Var}(k) I^{3}(k)=0 \text {. }
$$

Recall that $I^{3}(k)=0$ when $k$ is a $C_{2}$-field or, more generally, when $k$ is a field of cohomological dimension $\leq 2$. Examples include fields of transcendence degree $\leq 2$ over algebraically closed fields, $p$-adic fields, non formally real global fields, etc. 


\subsection{Products of quadrics}

Surprisingly, Theorem 2.15 admits the following generalization:

Theorem 2.19 Let $\left\{q_{j}\right\}_{1 \leq j \leq m}$ and $\left\{q_{j}^{\prime}\right\}_{1 \leq j \leq m^{\prime}}$ be two families of non-degenerate quadratic forms with trivial discriminant of dimension $n \geq 5$. If $\left[\Pi_{j=1}^{m} Q_{q_{j}}\right]=$ $\left[\Pi_{j=1}^{m^{\prime}} Q_{q_{j}^{\prime}}\right]$ in $K_{0} \operatorname{Var}(k)$, then the following holds:

(i) We have $\operatorname{dim}\left(\Pi_{j=1}^{m} Q_{q_{j}}\right)=\operatorname{dim}\left(\Pi_{j=1}^{m^{\prime}} Q_{q_{j}^{\prime}}\right)$. Equivalently, we have $m=m^{\prime}$.

(ii) We have $\left\langle\left\{\left[C_{0}^{+}\left(q_{j}\right)\right]\right\}_{j}\right\rangle=\left\langle\left\{\left[C_{0}^{+}\left(q_{j}^{\prime}\right)\right]\right\}_{j}\right\rangle$ when $n$ is even or $\left\langle\left\{\left[C_{0}\left(q_{j}\right)\right]\right\}_{j}\right\rangle=$ $\left\langle\left\{\left[C_{0}\left(q_{j}^{\prime}\right)\right]\right\}_{j}\right\rangle$ when $n$ is odd.

(iii) When $n=6$ and $m \leq 5$, we have $\Pi_{j=1}^{m} Q_{q_{j}} \simeq \Pi_{j=1}^{m} Q_{q_{j}^{\prime}}$.

(iv) When $I^{3}(k)=0$ and $m \leq 5$, we have $\Pi_{j=1}^{m} Q_{q_{j}} \simeq \Pi_{j=1}^{m} Q_{q_{j}^{\prime}}$.

(iv') When $I^{3}(k)=0, m \geq 6$, and the following extra condition holds (consult Notation 2.21 below)

$$
\begin{cases}\Sigma_{\text {even }}^{1}(m, n, l)>\Sigma_{\text {even }}^{2}(m, n, l) \text { for every } 2 \leq l \leq m-3 & \mathrm{n} \text { even } \\ \Sigma_{\text {odd }}^{1}(m, n, l)>\Sigma_{\text {odd }}^{2}(m, n, l) \text { for every } 2 \leq l \leq m-3 & \mathrm{n} \text { odd },\end{cases}
$$

we also have $\Pi_{j=1}^{m} Q_{q_{j}} \simeq \Pi_{j=1}^{m} Q_{q_{j}^{\prime}}$.

Notation 2.21 Given integers $m, n, l \geq 0$, consider the following sums of $(n-2)$ powers:

$$
\begin{aligned}
\Sigma_{\text {even }}^{1}(m, n, l):= & \Sigma_{r=0}^{\lfloor l / 2\rfloor}\left(\left(\begin{array}{c}
l \\
2 r
\end{array}\right) \times 2^{2 r+1} \times(n-2)^{m-(2 r+1)}+\left(\begin{array}{c}
l \\
2 r+1
\end{array}\right) \times 2^{m-l+(2 r+1)}\right. \\
& \left.\times(n-2)^{l-(2 r+1)}\right) \\
\Sigma_{\text {odd }}^{1}(m, n, l):= & \Sigma_{r=0}^{\lfloor l / 2\rfloor}\left(\left(\begin{array}{c}
l \\
2 r
\end{array}\right) \times(n-2)^{m-(2 r+1)}\right. \\
& \left.+\left(\begin{array}{c}
l \\
2 r+1
\end{array}\right) \times(n-2)^{l-(2 r+1)}\right) \\
\Sigma_{\text {even }}^{2}(m, n, l):= & \Sigma_{r=0}^{\lfloor l / 2\rfloor}\left(\left(\begin{array}{c}
l \\
2 r
\end{array}\right) \times 2^{2 r+2} \times(n-2)^{m-(2 r+2)}+\left(\begin{array}{c}
l \\
2 r+1
\end{array}\right) \times 2^{2 r+1}\right. \\
& \left.\times(n-2)^{m-(2 r+2)}\right) \\
\Sigma_{\text {odd }}^{2}(m, n, l):= & \Sigma_{r=0}^{\lfloor l / 2\rfloor}\left(\left(\begin{array}{c}
l \\
2 r
\end{array}\right) \times(n-2)^{m-(2 r+2)}\right. \\
& \left.+\left(\begin{array}{c}
l \\
2 r+1
\end{array}\right) \times(n-2)^{m-(2 r+2)}\right) .
\end{aligned}
$$

Note that item (iii) shows that when the dimension is equal to 6 , two products of quadrics (with $m \leq 5$ ) have the same Grothendieck class in $K_{0} \operatorname{Var}(k)$ if and only if they are isomorphic! This implies that the above inclusion (2.16) holds more generally for products of quadrics (with $m \leq 5$ ). In the same vein, items (iv-iv') show that when 
$I^{3}(k)=0$, two products of quadrics have the same Grothendieck class in $K_{0} \operatorname{Var}(k)$ if and only if they are isomorphic! This implies that the above inclusion (2.18) holds more generally for products of quadrics. Finally, note that the extra condition (2.20) holds whenever the dimension $n$ is $\gg$ than the number $m$ of quadrics because the highest power of $(n-2)$ in the sums $\Sigma_{\text {even }}^{1}(m, n, l)$ and $\Sigma_{\text {odd }}^{1}(m, n, l)$ is $(n-2)^{m-1}$ while the highest power of $(n-2)$ in the sums $\Sigma_{\text {even }}^{2}(m, n, l)$ and $\Sigma_{\text {odd }}^{2}(m, n, l)$ is $(n-2)^{m-2}$.

\subsection{Involution varieties}

Let $G$ be the projective special orthogonal group $P S O_{n}$, with $n \geq 6$ even, with respect to the hyperbolic form $\frac{n}{2} \mathbb{H}$. In this case, we have $\widetilde{G}=\operatorname{Spin}_{n}$. Similarly to $\S 2.4$, consider the projective homogeneous variety $\mathcal{F}$ given by $Q:=\left(x_{1} y_{1}+\cdots+x_{\frac{n}{2}} y_{\frac{n}{2}}=\right.$ $0) \subset \mathbb{P}^{n-1}$ and recall from loc. cit. that $R(\widetilde{P})=\oplus_{i=0}^{n-1} R(\widetilde{G}) \rho_{i}$. Given a 1-cocycle $\gamma: \Gamma \rightarrow P S O_{n}(\bar{k})$, let $(A, *)$ be the corresponding central simple $k$-algebra of degree $n$ with involution of orthogonal type and trivial discriminant. Under these notations, the twisted form ${ }_{\gamma} Q$ is the involution variety $\operatorname{Iv}(A, *) \subset \mathbb{P}^{n-1}$ and the Tits central simple $k$-algebra $A_{\rho_{i}}$ is given as follows

$$
A_{\rho_{i}}:= \begin{cases}k & 0 \leq i \leq n-3 \quad i \text { even } \\ A & 0 \leq i \leq n-3 \quad i \text { odd } \\ C_{0}^{+}(A, *) & i=n-2 \\ C_{0}^{-}(A, *) & i=n-1,\end{cases}
$$

where $C_{0}^{+}(A, *)$ and $C_{0}^{-}(A, *)$ stand for the simple components of the even Clifford algebra $C_{0}(A, *)$ of $(A, *)$.

Remark 2.22 (Generalization) In the particular case where $(A, *)$ is split, i.e., isomorphic to $\left(M_{n}(k), *_{q}\right)$ with $*_{q}$ the adjoint involution associated to a quadratic form $q$, the involution variety $\operatorname{Iv}(A, *) \subset \mathbb{P}^{n-1}$ reduces to the quadric hypersurface $Q_{q} \subset \mathbb{P}^{n-1}$. Hence, involution varieties may be understood as "forms of quadrics".

Theorem 2.23 Let $(A, *)$ and $\left(A^{\prime}, *^{\prime}\right)$ be two central simple $k$-algebras with involutions of orthogonal type and trivial discriminant. If $[\operatorname{Iv}(A, *)]=\left[\operatorname{Iv}\left(A^{\prime}, *^{\prime}\right)\right]$ in $K_{0} \operatorname{Var}(k)$, then the following holds:

(i) We have $\operatorname{dim}(\operatorname{Iv}(A, *))=\operatorname{dim}\left(\operatorname{Iv}\left(A^{\prime}, *^{\prime}\right)\right)$. Equivalently, we have $\operatorname{deg}(A)=$ $\operatorname{deg}\left(A^{\prime}\right)$.

(ii) We have $^{2} C_{0}^{ \pm}(A, *) \simeq C_{0}^{ \pm}\left(A^{\prime}, *^{\prime}\right)$.

(iii) When $\operatorname{deg}(A)=6$, we have $\operatorname{Iv}(A, *) \simeq \operatorname{Iv}\left(A^{\prime}, *^{\prime}\right)$.

(iv) When $I^{3}(k)=0$, we have $\operatorname{Iv}(A, *) \simeq \operatorname{Iv}\left(A^{\prime}, *^{\prime}\right)$.

2 The short notation $C_{0}^{ \pm}(A, *) \simeq C_{0}^{ \pm}\left(A^{\prime}, *^{\prime}\right)$ stands for $\left\{\begin{array}{l}C_{0}^{+}(A, *) \simeq C_{0}^{+}\left(A^{\prime}, *^{\prime}\right) \\ C_{0}^{-}(A, *) \simeq C_{0}^{-}\left(A^{\prime}, *^{\prime}\right)\end{array}\right.$ or $\left\{\begin{array}{l}C_{0}^{+}(A, *) \simeq C_{0}^{-}\left(A^{\prime}, *^{\prime}\right) \\ C_{0}^{-}(A, *) \simeq C_{0}^{+}\left(A^{\prime}, *^{\prime}\right) .\end{array}\right.$ 
Note that, similarly to Theorem 2.15 , item (iii), resp. (iv), shows that when the degree is equal to 6 , resp. $I^{3}(k)=0$, two involution varieties have the same Grothendieck class in $K_{0} \operatorname{Var}(k)$ if and only if they are isomorphic! In other words, items (iii)-(iv) yield the following inclusions:

$$
\frac{\{\text { Involution varieties } \operatorname{Iv}(A, *) \text { with trivial discriminant and } \operatorname{deg}(A)=6\}}{\text { isomorphism }} \subset K_{0} \operatorname{Var}(k)
$$

$$
\frac{\{\text { Involution varieties } \operatorname{Iv}(A, *) \text { with trivial discriminant }\}}{\text { isomorphism }} \subset K_{0} \operatorname{Var}(k) I^{3}(k)=0 \text {. }
$$

Following Remark 2.22, note that (2.24), resp. (2.25), extends the above inclusion (2.16), resp. (2.18).

Remark 2.26 (Dimension) Let $X$ and $Y$ be two varieties. As mentioned by one of the anonymous referees, it follows from the existence of the virtual Betti polynomial that if $[X]=[Y]$ in the Grothendieck ring of varieties $K_{0} \operatorname{Var}(k), \operatorname{then}^{3} \operatorname{dim}(X)=\operatorname{dim}(Y)$. This shows that item (i) of the above Theorems 2.2, 2.13, 2.15, 2.19 and 2.23 holds in much greater generality.

\section{Preliminaries}

Throughout the article $k$ denotes a base field of characteristic zero.

Notation 3.1 Throughout the article we will write $\oplus_{p} \operatorname{Br}(k)\{p\}$ for the $p$-primary decomposition of the Brauer group $\operatorname{Br}(k)$. Given a central simple $k$-algebra $A$ and a prime number $q$, let us write $[A]^{q} \in \operatorname{Br}(k)\{q\}$ for the $q$-primary component of the Brauer class $[A] \in \operatorname{Br}(k)=\oplus_{p} \operatorname{Br}(k)\{p\}$.

\section{Dg categories}

A differential graded $(=d g)$ category $\mathcal{A}$ is a category enriched over complexes of $k$ vector spaces; consult Keller's survey [11] (and Bondal-Kapranov's original article [4]). Every (dg) $k$-algebra $A$ gives naturally rise to a dg category with a single object. Another source of examples is provided by schemes since the category of perfect complexes perf $(X)$ of every $k$-scheme $X$ admits a canonical dg enhancement $\operatorname{perf}_{\mathrm{dg}}(X)$; consult $[11, \S 4.6]$. Let us denote by $\operatorname{dgcat}(k)$ the category of (small) dg categories.

Let $\mathcal{A}$ be a dg category. The opposite dg category $\mathcal{A}^{\text {op }}$ has the same objects as $\mathcal{A}$ and $\mathcal{A}^{\mathrm{op}}(x, y):=\mathcal{A}(y, x)$. A right $d g \mathcal{A}$-module is a dg functor $M: \mathcal{A}^{\mathrm{op}} \rightarrow \mathcal{C}_{\mathrm{dg}}(k)$ with values in the dg category of complexes of $k$-vector spaces. Let us denote by $\mathcal{C}(\mathcal{A})$ the category of right $\operatorname{dg} \mathcal{A}$-modules. Following $[11, \S 3.2]$, the derived category $\mathcal{D}(\mathcal{A})$ of $\mathcal{A}$ is defined as the localization of $\mathcal{C}(\mathcal{A})$ with respect to the objectwise quasiisomorphisms. In what follows, we will write $\mathcal{D}_{c}(\mathcal{A})$ for the subcategory of compact objects.

\footnotetext{
3 The implication $[X]=[Y] \Rightarrow \operatorname{dim}(X)=\operatorname{dim}(Y)$ holds similarly in positive characteristic.
} 
A dg functor $F: \mathcal{A} \rightarrow \mathcal{B}$ is called a Morita equivalence if it induces an equivalence on derived categories $\mathcal{D}(\mathcal{A}) \simeq \mathcal{D}(\mathcal{B})$; consult [11, §4.6]. As explained in [26, §1.6.1], dgcat $(k)$ admits a Quillen model structure whose weak equivalences are the Morita equivalences. Let $\operatorname{Hmo}(k)$ be the associated homotopy category.

The tensor product $\mathcal{A} \otimes \mathcal{B}$ of dg categories is defined as follows: the set of objects is the cartesian product of the sets of objects and $(\mathcal{A} \otimes \mathcal{B})((x, w),(y, z)):=$ $\mathcal{A}(x, y) \otimes \mathcal{B}(w, z)$. As explained in $[11, \S 2.3]$, this construction gives rise to a symmetric monoidal structure on $\operatorname{dgcat}(k)$ which descends to the homotopy category $\operatorname{Hmo}(k)$.

A $d g \mathcal{A}$ - $\mathcal{B}$-bimodule is a dg functor $\mathrm{B}: \mathcal{A} \otimes \mathcal{B}^{\mathrm{op}} \rightarrow \mathcal{C}_{\mathrm{dg}}(k)$. For example, given a $\mathrm{dg}$ functor $F: \mathcal{A} \rightarrow \mathcal{B}$, we have the $\operatorname{dg} \mathcal{A}$ - $\mathcal{B}$-bimodule ${ }_{F} \mathcal{B}: \mathcal{A} \otimes \mathcal{B}^{\text {op }} \rightarrow \mathcal{C}_{\mathrm{dg}}(k),(x, z) \mapsto$ $\mathcal{B}(z, F(x))$. Let $\operatorname{rep}(\mathcal{A}, \mathcal{B})$ be the full triangulated subcategory of $\mathcal{D}\left(\mathcal{A}^{\text {op }} \otimes \mathcal{B}\right)$ consisting of those $\mathrm{dg} \mathcal{A}$ - $\mathcal{B}$-bimodules $\mathrm{B}$ such that for every object $x \in \mathcal{A}$ the right $\mathrm{dg}$ $\mathcal{B}$-module $\mathrm{B}(x,-)$ belongs to $\mathcal{D}_{c}(\mathcal{B})$. Clearly, the $\operatorname{dg} \mathcal{A}$ - $\mathcal{B}$-bimodules ${ }_{F} \mathcal{B}$ belongs to $\operatorname{rep}(\mathcal{A}, \mathcal{B})$.

Finally, following Kontsevich [15-17], a $\operatorname{dg}$ category $\mathcal{A}$ is called smooth if the $\mathrm{dg}$ $\mathcal{A}-\mathcal{A}$ bimodule id $\mathcal{A}$ belongs to $\mathcal{D}_{c}\left(\mathcal{A}^{\text {op }} \otimes \mathcal{A}\right)$ and proper if $\Sigma_{n} \operatorname{dim} H^{n} \mathcal{A}(x, y)<\infty$ for any pair of objects $(x, y)$. Examples include finite-dimensional $k$-algebras of finite global dimension $A$ as well as the dg categories of perfect complexes $\operatorname{perf}_{\mathrm{dg}}(X)$ associated to smooth proper $k$-schemes $X$.

\section{Noncommutative motives}

For a book on noncommutative motives, we invite the reader to consult [26]. As explained in [26, §1.6.3], given any two $\operatorname{dg}$ categories $\mathcal{A}$ and $\mathcal{B}$, there is a natural bijection between $\operatorname{Hom}_{\operatorname{Hmo}(k)}(\mathcal{A}, \mathcal{B})$ and the set of isomorphism classes of the category $\operatorname{rep}(\mathcal{A}, \mathcal{B})$. Under this bijection, the composition in $\operatorname{Hmo}(k)$ corresponds to the (derived) tensor product of bimodules. The additivization of $\operatorname{Hmo}(k)$ is the additive category $\mathrm{Hmo}_{0}(k)$ with the same objects and with abelian groups of morphisms $\operatorname{Hom}_{\mathrm{Hmo}_{0}(k)}(\mathcal{A}, \mathcal{B})$ given by the Grothendieck group $K_{0} \operatorname{rep}(\mathcal{A}, \mathcal{B})$ of the triangulated category $\operatorname{rep}(\mathcal{A}, \mathcal{B})$. The composition law is induced by the (derived) tensor product of bimodules. Given a commutative ring of coefficients $R$, the $R$-linearization of $\mathrm{Hmo}_{0}(k)$ is the $R$-linear category $\operatorname{Hmo}_{0}(k)_{R}$ obtained by tensoring the morphisms of $\mathrm{Hmo}_{0}(k)$ with $R$. Note that we have the following (composed) symmetric monoidal functor

$$
U(-)_{R}: \operatorname{dgcat}(k) \longrightarrow \mathrm{Hmo}_{0}(k)_{R} \mathcal{A} \mapsto \mathcal{A}(\mathcal{A} \stackrel{F}{\rightarrow} \mathcal{B}) \mapsto\left[{ }_{F} \mathcal{B}\right]_{R}
$$

The category of noncommutative Chow motives $\operatorname{NChow}(k)_{R}$ is defined as the idempotent completion of the full subcategory of $\operatorname{Hmo}_{0}(k)_{R}$ consisting of the objects $U(\mathcal{A})_{R}$ with $\mathcal{A}$ a smooth proper dg category. This category is $R$-linear, additive, rigid symmetric monoidal, and idempotent complete. When $R=\mathbb{Z}$, we will write $\operatorname{NChow}(k)$ instead of $\operatorname{NChow}(k)_{\mathbb{Z}}$ and $U(-)$ instead of $U(-)_{\mathbb{Z}}$.

Given an additive rigid symmetric monoidal category $\mathcal{C}$, recall that its $\mathcal{N}$-ideal is defined as follows 
$\mathcal{N}(a, b):=\left\{f \in \operatorname{Hom}_{\mathcal{C}}(a, b) \mid \forall g \in \operatorname{Hom}_{\mathcal{C}}(b, a)\right.$ we have $\left.\operatorname{tr}(g \circ f)=0\right\}$,

where $\operatorname{tr}(g \circ f)$ stands for the categorical trace of $g \circ f$. The category of noncommutative numerical motives $\mathrm{NNum}(k)_{R}$ is defined as the idempotent completion of the quotient of $\operatorname{NChow}(k)_{R}$ by the $\otimes$-ideal $\mathcal{N}$. By construction, this category is $R$-linear, additive, rigid symmetric monoidal, and idempotent complete.

Remark 3.2 (Smooth proper schemes) Given any two smooth projective $k$-schemes $X$ and $Y$, we have the following Morita equivalence (consult [29, Lem. 4.26]):

$$
\operatorname{perf}_{\mathrm{dg}}(X) \otimes \operatorname{perf}_{\mathrm{dg}}(Y) \longrightarrow \operatorname{perf}_{\mathrm{dg}}(X \times Y)(\mathcal{F}, \mathcal{G}) \mapsto \mathcal{F} \otimes \mathcal{G}
$$

Using the fact that the functor $U(-)_{R}$ is symmetric monoidal, we hence conclude that the noncommutative (Chow or numerical) motives $U\left(\operatorname{perf}_{\mathrm{dg}}(X \times Y)\right)_{R}$ and $U\left(\operatorname{perf}_{\mathrm{dg}}(X)\right)_{R} \otimes U\left(\operatorname{perf}_{\mathrm{dg}}(Y)\right)_{R}$ are isomorphic.

\section{Proof of Theorem 1.2}

Let $K_{0}(\mathrm{NChow}(k))$ be the Grothendieck ring of the additive symmetric monoidal category of noncommutative Chow motives $\operatorname{NChow}(k)$. We start by constructing a motivic measure with values in this ring.

Proposition 4.1 The assignment $X \mapsto U\left(\operatorname{perf}_{\mathrm{dg}}(X)\right)$, with $X$ a smooth projective $k$-scheme, gives rise to a motivic measure $\mu_{\mathrm{nc}}: K_{0} \operatorname{Var}(k) \rightarrow K_{0}(\operatorname{NChow}(k))$.

Proof Thanks to Bittner's presentation [3, Thm. 3.1] of the Grothendieck ring of varieties $K_{0} \operatorname{Var}(k)$, it suffices to prove the following two conditions:

(i) Let $X$ be a smooth projective $k$-scheme, $Y \hookrightarrow X$ a smooth closed subscheme of codimension $c, \mathrm{Bl}_{Y}(X)$ the blow-up of $X$ along $Y$, and $E$ the exceptional divisor of the blow-up. Under these notations, we have the following equality in the Grothendieck ring $K_{0}(\operatorname{NChow}(k))$ :

$$
\begin{aligned}
& {\left[U\left(\operatorname{perf}_{\mathrm{dg}}\left(\mathrm{Bl}_{Y}(X)\right)\right)\right]-\left[U\left(\operatorname{perf}_{\mathrm{dg}}(E)\right)\right]} \\
& \quad=\left[U\left(\operatorname{perf}_{\mathrm{dg}}(X)\right)\right]-\left[U\left(\operatorname{perf}_{\mathrm{dg}}(Y)\right)\right] .
\end{aligned}
$$

(ii) Given smooth projective $k$-schemes $X$ and $Y$, we have the following equality in $K_{0}(\mathrm{NChow}(k))$ :

$$
\left[U\left(\operatorname{perf}_{\mathrm{dg}}(X \times Y)\right)\right]=\left[U\left(\operatorname{perf}_{\mathrm{dg}}(X)\right) \otimes U\left(\operatorname{perf}_{\mathrm{dg}}(Y)\right)\right]
$$

Let us write $f: \mathrm{Bl}_{Y}(X) \rightarrow X$ for the blow-up map, $i: E \hookrightarrow \mathrm{Bl}_{Y}(X)$ for the embedding map, and $p: E \rightarrow Y$ for the projection map (= restriction of $f$ to $E$ ). Under these 
notations, recall from Orlov [21, Thm. 4.3] that we have the following semi-orthogonal decompositions

$$
\begin{aligned}
\operatorname{perf}\left(\operatorname{Bl}_{Y}(X)\right) & =\left\langle\mathbb{L} f^{*}(\operatorname{perf}(X)), \Psi_{0}(\operatorname{perf}(Y)), \ldots, \Psi_{c-2}(\operatorname{perf}(Y))\right\rangle \\
\operatorname{perf}(E) & =\left\langle\Phi_{0}(\operatorname{perf}(Y)), \ldots, \Phi_{c-1}(\operatorname{perf}(Y))\right\rangle,
\end{aligned}
$$

where $\Psi_{i}(-):=\mathbb{R} i_{*}\left(\mathbb{L} p^{*}(-) \otimes^{\mathbb{L}} \mathcal{O}_{E / Y}(i)\right)$ and $\Phi_{i}(-):=\mathbb{L} p^{*}(-) \otimes^{\mathbb{L}} \mathcal{O}_{E / Y}(i)$. Moreover, the functors $\mathbb{L} f^{*}(-), \Psi_{i}(-)$, and $\Phi_{i}(-)$, are fully-faithful and of FourierMukai type. Consequently, using the fact that the functor $U$ sends semi-orthogonal decomposition to direct sums (consult $[26, \S 2.1]$ ), we conclude that

$$
\begin{aligned}
{\left[U\left(\operatorname{perf}_{\mathrm{dg}}\left(\mathrm{Bl}_{Y}(X)\right)\right)\right]=} & {\left[U\left(\operatorname{perf}_{\mathrm{dg}}(X)\right)\right] } \\
& +(c-1)\left[U\left(\operatorname{perf}_{\mathrm{dg}}(Y)\right)\right] \text { and }\left[U\left(\operatorname{perf}_{\mathrm{dg}}(E)\right)\right] \\
= & c\left[U\left(\operatorname{perf}_{\mathrm{dg}}(Y)\right)\right] .
\end{aligned}
$$

These equalities imply condition (i). Condition (ii) follows now from the above Remark 3.2.

Notation 4.2 Given a commutative ring of coefficients $R$, let us write $\operatorname{CSA}(k)_{R}$ for the full subcategory of $\operatorname{NChow}(k)_{R}$ consisting of the objects $U(A)_{R}$ with $A$ a central simple $k$-algebra. The closure of $\operatorname{CSA}(k)_{R}$ under finite direct sums will be denoted by $\operatorname{CSA}(k)_{R}^{\oplus}$. In the same vein, let us write $\underline{\operatorname{CSA}}(k)_{R}$ for the full subcategory of $\operatorname{NNum}(k)_{R}$ consisting of the objects $U(A)_{R}$ with $A$ a central simple $k$-algebra, and $\underline{\mathrm{CSA}}(k)_{R}^{\oplus}$ for its closure under finite direct sums. In the particular case where $R=\mathbb{Z}$, we will omit the underscript $(-)_{\mathbb{Z}}$.

Proposition 4.3 Given two central simple $k$-algebras $A$ and $A^{\prime}$ and a prime number $p$, the following holds:

(i) We have an isomorphism $U(A)_{\mathbb{F}_{p}} \simeq U\left(A^{\prime}\right)_{\mathbb{F}_{p}}$ in the category $\operatorname{CSA}(k)_{\mathbb{F}_{p}}$ (or, equivalently, in the category $\left.\underline{\operatorname{CSA}}(k)_{\mathbb{F}_{p}}\right)$ if and only if $[A]^{p}=\left[A^{\prime}\right]^{p}$ in $\operatorname{Br}(k)\{p\}$.

(ii) The $\mathbb{F}_{p}$-vector space $\operatorname{Hom}_{\underline{C S A}(k)_{\mathbb{F}_{p}}}\left(U(A)_{\mathbb{F}_{p}}, U\left(A^{\prime}\right)_{\mathbb{F}_{p}}\right)$ is naturally isomorphic to $\begin{cases}\mathbb{F}_{p} & \text { when }[A]^{p}=\left[A^{\prime}\right]^{p} \\ 0 & \text { when }[A]^{p} \neq\left[A^{\prime}\right]^{p} .\end{cases}$

Proof Given central simple $k$-algebras $A, A^{\prime}, A^{\prime \prime}$, recall from the proof of [28, Prop. 2.26] that the following composition map

$$
\begin{aligned}
& \operatorname{Hom}_{\mathrm{CSA}(k)}\left(U(A), U\left(A^{\prime}\right)\right) \times \operatorname{Hom}_{\mathrm{CSA}(k)}\left(U\left(A^{\prime}\right), U\left(A^{\prime \prime}\right)\right) \\
& \longrightarrow \operatorname{Hom}_{\mathrm{CSA}(k)}\left(U(A), U\left(A^{\prime \prime}\right)\right)
\end{aligned}
$$

corresponds to the following bilinear pairing:

$$
\mathbb{Z} \times \mathbb{Z} \longrightarrow \mathbb{Z} \quad(n, m) \mapsto n \cdot \operatorname{ind}\left(A^{\mathrm{op}} \otimes A^{\prime}\right) \cdot \operatorname{ind}\left(A^{\prime \mathrm{op}} \otimes A^{\prime \prime}\right) \cdot m .
$$


Since ind $\left(A^{\mathrm{op}} \otimes A^{\prime}\right)=\operatorname{ind}\left(A^{\prime \mathrm{op}} \otimes A\right)$, this implies, in particular, that the composition map

$$
\begin{aligned}
& \operatorname{Hom}_{\mathrm{CSA}(k)_{\mathbb{F}_{p}}}\left(U(A)_{\mathbb{F}_{p}}, U\left(A^{\prime}\right)_{\mathbb{F}_{p}}\right) \times \operatorname{Hom}_{\mathrm{CSA}(k)_{\mathbb{F}}}\left(U\left(A^{\prime}\right)_{\mathbb{F}_{p}}, U(A)_{\mathbb{F}_{p}}\right) \\
& \longrightarrow \operatorname{Hom}_{\mathrm{CSA}(k)_{\mathbb{F}_{p}}}\left(U(A)_{\mathbb{F}_{p}}, U(A)_{\mathbb{F}_{p}}\right)
\end{aligned}
$$

corresponds to the following bilinear pairing

$$
\mathbb{F}_{p} \times \mathbb{F}_{p} \longrightarrow \mathbb{F}_{p}(n, m) \mapsto n \cdot \operatorname{ind}\left(A^{\text {op }} \otimes A^{\prime}\right)^{2} \cdot m
$$

similarly with $A$ and $A^{\prime}$ replaced by $A^{\prime}$ and $A$, respectively. On the one hand, if $p \mid \operatorname{ind}\left(A^{\mathrm{op}} \otimes A^{\prime}\right)$, the bilinear pairing (4.4) is equal to zero. This implies that $U(A)_{\mathbb{F}_{p}} \not U\left(A^{\prime}\right)_{\mathbb{F}_{p}}$ in the category $\operatorname{CSA}(k)_{\mathbb{F}_{p}}$ and, by definition of the

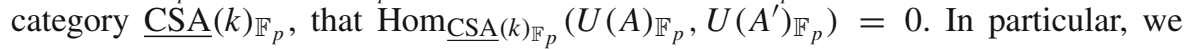
also have $U(A)_{\mathbb{F}_{p}} \not 千 U\left(A^{\prime}\right)_{\mathbb{F}_{p}}$ in the category $\underline{\operatorname{CSA}}(k)_{\mathbb{F}_{p}}$. On the other hand, if $p \nmid \operatorname{ind}\left(A^{\mathrm{op}} \otimes A^{\prime}\right)$, the integer ind $\left(A^{\mathrm{op}} \otimes A^{\prime}\right)$ is invertible in $\mathbb{F}_{p}$. Consequently, we conclude from (4.4) that $U(A)_{\mathbb{F}_{p}} \simeq U\left(A^{\prime}\right)_{\mathbb{F}_{p}}$ in the category $\operatorname{CSA}(k)_{\mathbb{F}_{p}}$ (and hence in the category $\left.\underline{\operatorname{CSA}}(k)_{\mathbb{F}_{p}}\right)$. Thanks to the definition of the category

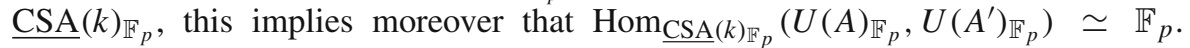
Finally, note that the $p$-primary decomposition of the Brauer group $\operatorname{Br}(k)=$ $\oplus_{p} \operatorname{Br}(k)\{p\}$ implies that $p \nmid \operatorname{ind}\left(A^{\text {op }} \otimes A^{\prime}\right)$ if and only if $[A]^{p}=\left[A^{\prime}\right]^{p}$ in $\operatorname{Br}(k)\{p\}$.

Note that Proposition 4.3 implies, in particular, the following result:

Corollary 4.5 Given a prime number $p$, we have an induced equivalence of categories

$$
\begin{aligned}
& \underline{\operatorname{CSA}}(k)_{\mathbb{F}_{p}}^{\oplus} \stackrel{\simeq}{\longrightarrow} \operatorname{Vect}_{\operatorname{Br}(k)\{p\}}(k) U\left(A_{1}\right)_{\mathbb{F}_{p}} \oplus \cdots \oplus U\left(A_{m}\right) \mathbb{F}_{p} \\
& \mapsto\left(\mathbb{F}_{p}\left[A_{1}\right]^{p}\right) \oplus \cdots \oplus\left(\mathbb{F}_{p}\left[A_{m}\right]^{p}\right),
\end{aligned}
$$

where $\operatorname{Vect} \operatorname{Br}_{(k)\{p\}}(k)$ stands for the category of finite-dimensional $\operatorname{Br}(k)\{p\}$-graded $\mathbb{F}$ p-vector spaces.

Recall from [28, Thm. 2.20(iv)] the following result:

Proposition 4.7 Given two families of central simple $k$-algebras $\left\{A_{j}\right\}_{1 \leq j \leq m}$ and $\left\{A_{j}^{\prime}\right\}_{1 \leq j \leq m^{\prime}}$, the following conditions are equivalent:

(i) We have an isomorphism $\oplus_{j=1}^{m} U\left(A_{j}\right) \simeq \oplus_{j=1}^{m^{\prime}} U\left(A_{j}^{\prime}\right)$ in the category $\operatorname{CSA}(k)^{\oplus}$.

(ii) We have $m=m^{\prime}$ and for every prime number $p$ there exists a permutation $\sigma_{p}$ (which depends on $p$ ) such that $\left[A_{j}^{\prime}\right]^{p}=\left[A_{\sigma_{p}(j)}\right]^{p}$ in $\operatorname{Br}(k)\{p\}$ for every $1 \leq j \leq$ $m$.

Corollary 4.8 Let $\left\{A_{j}\right\}_{1 \leq j \leq m}$ and $\left\{A_{j}^{\prime}\right\}_{1 \leq j \leq m^{\prime}}$ be two families of central simple $k$ algebras. If we have an isomorphism $\oplus_{j=1}^{m} U\left(A_{j}\right) \simeq \oplus_{j=1}^{m^{\prime}} U\left(A_{j}^{\prime}\right)$ in the category $\operatorname{CSA}(k)^{\oplus}$, then $\left\langle\left\{\left[A_{j}\right]\right\}_{1 \leq j \leq m}\right\rangle=\left\langle\left\{\left[A_{j}^{\prime}\right]\right\}_{1 \leq j \leq m^{\prime}}\right\rangle$. 
The following result is of independent interest:

Proposition 4.9 (Cancellation) Let $\left\{A_{j}\right\}_{1 \leq j \leq m}$ and $\left\{A_{j}^{\prime}\right\}_{1 \leq j \leq m^{\prime}}$ be two families of central simple $k$-algebras and $N M$ a noncommutative Chow motive. If $\oplus_{j=1}^{m} U\left(A_{j}\right) \oplus$ $N M \simeq \oplus_{j=1}^{m^{\prime}} U\left(A_{j}^{\prime}\right) \oplus N M$ in the category $\operatorname{NChow}(k)$, then $m=m^{\prime}$ and $\oplus_{j=1}^{m} U\left(A_{j}\right) \simeq$ $\oplus_{j=1}^{m} U\left(A_{j}^{\prime}\right)$.

Proof Given a (fixed) prime number $p$, consider the induced isomorphism

$$
\oplus_{j=1}^{m} U\left(A_{j}\right)_{\mathbb{F}_{p}} \oplus N M_{\mathbb{F}_{p}} \simeq \oplus_{j=1}^{m^{\prime}} U\left(A_{j}^{\prime}\right)_{\mathbb{F}_{p}} \oplus N M_{\mathbb{F}_{p}}
$$

in the category $\operatorname{NNum}(k)_{\mathbb{F}_{p}}$. Thanks to Lemma 4.15 below, there exists a noncommutative numerical motive $M N$ and integers $r_{j}, r_{j}^{\prime} \geq 0$ such that $N M_{\mathbb{F}_{p}} \simeq$ $\oplus_{j=1}^{m} U\left(A_{j}\right)_{\mathbb{F}_{p}}^{\oplus r_{j}} \oplus \oplus_{j=1}^{m^{\prime}} U\left(A_{j}^{\prime}\right)_{\mathbb{F}_{p}}^{\oplus r_{j}^{\prime}} \oplus M N$ in the category $\operatorname{NNum}(k)_{\mathbb{F}_{p}}$. Moreover, $M N$ does not contains the noncommutative numerical motives $\left\{U\left(A_{j}\right)_{\mathbb{F}_{p}}\right\}_{1 \leq j \leq m}$ and $\left\{U\left(A_{j}^{\prime}\right)_{\mathbb{F}_{p}}\right\}_{1 \leq j \leq m^{\prime}}$ as direct summands. Consequently, (4.10) yields an isomorphism:

$$
\begin{aligned}
& \oplus_{j=1}^{m} U\left(A_{j}\right)_{\mathbb{F}_{p}}^{\oplus\left(r_{j}+1\right)} \oplus \oplus_{j=1}^{m^{\prime}} U\left(A_{j}^{\prime}\right)_{\mathbb{F}_{p}}^{\oplus r_{j}^{\prime}} \oplus M N \\
& \simeq \oplus_{j=1}^{m} U\left(A_{j}\right)_{\mathbb{F}_{p}}^{\oplus r_{j}} \oplus \oplus_{j=1}^{m^{\prime}} U\left(A_{j}^{\prime}\right)_{\mathbb{F}_{p}}^{\oplus\left(r_{j}^{\prime}+1\right)} \oplus M N .
\end{aligned}
$$

We claim that the following composition maps (with $1 \leq i \leq m$ and $1 \leq j \leq m^{\prime}$ )

$$
\begin{aligned}
& \operatorname{Hom}_{\mathrm{NNum}(k)_{\mathbb{F}}}\left(U\left(A_{i}\right)_{\mathbb{F}_{p}}, M N\right) \times \operatorname{Hom}_{\mathrm{NNum}(k)_{\mathbb{F}_{p}}}\left(M N, U\left(A_{j}^{\prime}\right)_{\mathbb{F}_{p}}\right) \\
& \rightarrow \operatorname{Hom}_{\mathrm{NNum}(k)_{\mathbb{F}_{p}}}\left(U\left(A_{i}\right)_{\mathbb{F}_{p}}, U\left(A_{j}^{\prime}\right)_{\mathbb{F}_{p}}\right)
\end{aligned}
$$

are equal to zero; similarly with $A_{i}$ and $A_{j}^{\prime}$ replaced by $A_{j}^{\prime}$ and $A_{i}$, respectively. On the one hand, if $\left[A_{i}\right]^{p} \neq\left[A_{j}^{\prime}\right]^{p}$, it follows from the above Proposition 4.3(ii) that the right-hand side of (4.12) is equal to zero. On the other hand, if $\left[A_{i}\right]^{p}=\left[A_{j}^{\prime}\right]^{p}$, it follows Proposition 4.3 that $U\left(A_{i}\right)_{\mathbb{F}_{p}} \simeq U\left(A_{j}^{\prime}\right)_{\mathbb{F}_{p}}$ in the category $\operatorname{NNum}(k)_{\mathbb{F}_{p}}$ and that the right-hand side of (4.12) is isomorphic to $\mathbb{F}_{p}$. Since the category $\operatorname{NNum}(k)_{\mathbb{F}_{p}}$ is $\mathbb{F}_{p}$-linear and $M N$ does not contains the noncommutative numerical motives $\left\{U\left(A_{j}\right)_{\mathbb{F}_{p}}\right\}_{1 \leq j \leq m}$ and $\left\{U\left(A_{j}^{\prime}\right)_{\mathbb{F}_{p}}\right\}_{1 \leq j \leq m^{\prime}}$ as direct summands, we then conclude that the composition map (4.12) is necessarily equal to zero; otherwise $M N$ would contain $U\left(A_{i}\right)_{\mathbb{F}_{p}}$, or equivalently $U\left(A_{j}^{\prime}\right)_{\mathbb{F}_{p}}$, as a direct summand.

Note that the triviality of the composition maps (4.12) implies that the above isomorphism (4.11) in the category $\operatorname{NNum}(k)_{\mathbb{F}_{p}}$ restricts to an isomorphism

$$
\oplus_{j=1}^{m} U\left(A_{j}\right)_{\mathbb{F}_{p}}^{\oplus\left(r_{j}+1\right)} \oplus \oplus_{j=1}^{m^{\prime}} U\left(A_{j}^{\prime}\right)_{\mathbb{F}_{p}}^{\oplus r_{j}^{\prime}} \simeq \oplus_{j=1}^{m} U\left(A_{j}\right)_{\mathbb{F}_{p}}^{\oplus r_{j}} \oplus \oplus_{j=1}^{m^{\prime}} U\left(A_{j}^{\prime}\right)_{\mathbb{F}_{p}}^{\oplus\left(r_{j}^{\prime}+1\right)}
$$


in the full subcategory $\underline{\operatorname{CSA}}(k)_{\mathbb{F}_{p}}^{\oplus}$. Recall from Corollary 4.5 that the category $\underline{\mathrm{CSA}}(k)_{\mathbb{F}_{p}}^{\oplus}$ is equivalent to the category $\operatorname{Vect} \operatorname{Br}(k)\{p\}(k)$ of finite-dimensional $\operatorname{Br}(k)\{p\}$ graded $\mathbb{F}_{p}$-vector spaces. Under the equivalence (4.6), the isomorphism (4.13) corresponds to the following isomorphism:

$$
\begin{aligned}
& \oplus_{j=1}^{m}\left(\mathbb{F}_{p}^{\oplus\left(r_{j}+1\right)}\left[A_{j}\right]^{p}\right) \oplus \oplus_{j=1}^{m^{\prime}}\left(\mathbb{F}_{p}^{\oplus r_{j}^{\prime}}\left[A_{j}^{\prime}\right]^{p}\right) \\
& \simeq \oplus_{j=1}^{m}\left(\mathbb{F}_{p}^{\oplus r_{j}}\left[A_{j}\right]^{p}\right) \oplus \oplus_{j=1}^{m^{\prime}}\left(\mathbb{F}_{p}^{\oplus\left(r_{j}^{\prime}+1\right)}\left[A_{j}^{\prime}\right]^{p}\right) .
\end{aligned}
$$

Therefore, since the category $\operatorname{Vect}_{\operatorname{Br}(k)\{p\}}(k)$ has the Krull-Schmidt property, it follows from (4.14) that the finite-dimensional $\operatorname{Br}(k)\{p\}$-graded $\mathbb{F}_{p}$-vector spaces $\oplus_{j=1}^{m}\left(\mathbb{F}_{p}\left[A_{j}\right]^{p}\right)$ and $\oplus_{j=1}^{m^{\prime}}\left(\mathbb{F}_{p}\left[A_{j}^{\prime}\right]^{p}\right)$ are isomorphic, that $m=m^{\prime}$, and that there exists a permutation $\sigma_{p}$ (which depends on $p$ ) such that $\left[A_{j}^{\prime}\right]^{p}=\left[A_{\sigma_{p}(j)}\right]^{p}$ in $\operatorname{Br}(k)\{p\}$ for every $1 \leq j \leq m$. Consequently, the proof follows now from Proposition 4.7.

Lemma 4.15 We have $N M_{\mathbb{F}_{p}} \simeq \oplus_{j=1}^{m} U\left(A_{j}\right)_{\mathbb{F}_{p}}^{\oplus r_{j}} \oplus \oplus_{j=1}^{m^{\prime}} U\left(A_{j}^{\prime}\right)_{\mathbb{F}_{p}}^{\oplus r_{j}^{\prime}} \oplus M N$ in the category $\operatorname{NNum}(k)_{\mathbb{F}_{p}}$ for some noncommutative numerical motive $M N$ and integers $r_{j}, r_{j}^{\prime} \geq 0$. Moreover, $M N$ does not contains the noncommutative numerical motives $\left\{U\left(A_{j}\right)_{\mathbb{F}_{p}}\right\}_{1 \leq j \leq m}$ and $\left\{U\left(A_{j}^{\prime}\right)_{\mathbb{F}_{p}}\right\}_{1 \leq j \leq m^{\prime}}$ as direct summands.

Proof By definition, the category $\operatorname{NNum}(k)_{\mathbb{F}_{p}}$ is idempotent complete. Therefore, by inductively splitting all the (possible) direct summands $\left\{U\left(A_{j}\right)_{\mathbb{F}_{p}}\right\}_{1 \leq j \leq m}$ and $\left\{U\left(A_{j}^{\prime}\right)_{\mathbb{F}_{p}}\right\}_{1 \leq j \leq m^{\prime}}$ of $N M_{\mathbb{F}_{p}}$, we obtain an isomorphism

$$
N M_{\mathbb{F}_{p}} \simeq \oplus_{j=1}^{m} U\left(A_{j}\right)_{\mathbb{F}_{p}}^{\oplus r_{j}} \oplus \oplus_{j=1}^{m^{\prime}} U\left(A_{j}^{\prime}\right)_{\mathbb{F}_{p}}^{\oplus r_{j}^{\prime}} \oplus M N
$$

in the category $\operatorname{NNum}(k)_{\mathbb{F}_{p}}$ for some noncommutative numerical motive $M N$ and integers $r_{j}, r_{j}^{\prime} \geq 0$. Note that the inductive splitting procedure stops at a finite stage. Otherwise, the following $\mathbb{F}_{p}$-vector spaces

$$
\operatorname{Hom}_{\mathrm{NNum}(k)_{\mathbb{F}}}\left(U\left(A_{i}\right)_{\mathbb{F}_{p}}, N M_{\mathbb{F}_{p}}\right) \operatorname{Hom}_{\mathrm{NNum}(k)_{\mathbb{F}_{p}}}\left(U\left(A_{j}^{\prime}\right)_{\mathbb{F}_{p}}, N M_{\mathbb{F}_{p}}\right)
$$

would be infinite-dimensional, which is impossible because the $\mathbb{F}_{p}$-vector spaces $\operatorname{Hom}_{\mathrm{NNum}(k)}\left(U\left(A_{i}\right), N M\right) \otimes \mathbb{F}_{p}$ and $\operatorname{Hom}_{\mathrm{NNum}(k)}\left(U\left(A_{j}^{\prime}\right), N M\right) \otimes \mathbb{F}_{p}$ are finitedimensional (consult $[27, \S 6])$ and surject onto (4.17).

Notation 4.18 Let us write $K_{0}\left(\operatorname{CSA}(k)^{\oplus}\right)$ for the Grothendieck ring of the additive symmetric monoidal category $\operatorname{CSA}(k)^{\oplus}$; consult Notation 4.2.

Proposition 4.19 The inclusion of categories $\operatorname{CSA}(k)^{\oplus} \subset \mathrm{NChow}(k)$ gives rise to an injective ring homomorphism $K_{0}\left(\mathrm{CSA}(k)^{\oplus}\right) \rightarrow K_{0}(\operatorname{NChow}(k))$.

Proof Recall first that the group completion of an arbitrary monoid $(M,+)$ is defined as the quotient of the product $M \times M$ by the following equivalence relation:

$$
(m, n) \sim\left(m^{\prime}, n^{\prime}\right):=\exists r \in M \text { such that } m+n^{\prime}+r=n+m^{\prime}+r .
$$


Let us write $K_{0}(\operatorname{NChow}(k))^{+}$for the semi-ring of the additive symmetric monoidal category $\operatorname{NChow}(k)$. Concretely, $K_{0}(\operatorname{NChow}(k))^{+}$is the set of isomorphism classes of noncommutative Chow motives equipped with the addition, resp. multiplication, law induced by $\oplus$, resp. $\otimes$. In the same vein, let us write $K_{0}\left(\operatorname{CSA}(k)^{\oplus}\right)^{+}$for the semi-ring of the additive symmetric monoidal category $\operatorname{CSA}(k)^{\oplus}$. Clearly, the inclusion of categories $\operatorname{CSA}(k)^{\oplus} \subset \operatorname{NChow}(k)$ gives rise to an injective homomorphism $K_{0}\left(\mathrm{CSA}(k)^{\oplus}\right)^{+} \rightarrow K_{0}(\mathrm{NChow}(k))^{+}$. Therefore, by combining the above definition of group completion (4.20) with the cancellation Proposition 4.19, we conclude that the induced (ring) homomorphism $K_{0}\left(\operatorname{CSA}(k)^{\oplus}\right) \rightarrow K_{0}(\operatorname{NChow}(k))$ is also injective.

Consider the (composed) ring homomorphism

$$
K_{0} \operatorname{Var}(k)^{\mathrm{tw}} \subset K_{0} \operatorname{Var}(k) \stackrel{\mu_{\mathrm{nc}}}{\longrightarrow} K_{0}(\operatorname{NChow}(k)) .
$$

Let $\gamma \mathcal{F}$ be a twisted projective homogeneous variety with Tits central simple

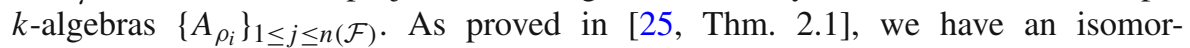
phism $U\left(\operatorname{perf}_{\mathrm{dg}}(\gamma \mathcal{F})\right) \simeq \oplus_{i=1}^{n(\mathcal{F})} U\left(A_{\rho_{i}}\right)$ in $\operatorname{NChow}(k)$. Therefore, making use of Proposition 4.19, we conclude that the assignment $\left[{ }_{\gamma} \mathcal{F}\right] \mapsto\left[\oplus_{i=1}^{n(\mathcal{F})} U\left(A_{\rho_{i}}\right)\right]=$ $\Sigma_{i=1}^{n(\mathcal{F})}\left[U\left(A_{\rho_{i}}\right)\right]$ gives rise to a (well-defined) motivic measure $K_{0} \operatorname{Var}(k)^{\mathrm{tw}} \rightarrow$ $K_{0}\left(\operatorname{CSA}(k)^{\oplus}\right)$. Consequently, the proof of Theorem 1.2 follows now from the following result:

Proposition 4.22 The assignment $[A] \mapsto[U(A)]$ gives rise to a ring isomorphism $R_{\mathrm{B}}(k) \stackrel{\simeq}{\rightarrow} K_{0}\left(\mathrm{CSA}(k)^{\oplus}\right)$.

Proof Let us write $K_{0}\left(\mathrm{CSA}(k)^{\oplus}\right)^{+}$for the semi-ring of the additive symmetric monoidal category $\operatorname{CSA}(k)^{\oplus}$. Concretely, $K_{0}\left(\operatorname{CSA}(k)^{\oplus}\right)^{+}$is the set of isomorphism classes of the category $\operatorname{CSA}(k)^{\oplus}$ equipped with the addition, resp. multiplication, law induced by $\oplus$, resp. $\otimes$. Consider also the semi-ring $\mathbb{N}[\operatorname{Br}(k)]$ and the following (semi-ring) homomorphism:

$$
\mathbb{N}[\operatorname{Br}(k)] \longrightarrow K_{0}\left(\operatorname{CSA}(k)^{\oplus}\right)^{+} \Sigma_{j=1}^{m}\left[A_{j}\right] \mapsto \Sigma_{j=1}^{m}\left[U\left(A_{j}\right)\right] .
$$

The homomorphism (4.23) is surjective. Moreover, thanks to Proposition 4.7, it yields an isomorphism

$$
\begin{aligned}
& \mathbb{N}[\operatorname{Br}(k)] /\left\{\sum_{j=1}^{m}\left[A_{j}\right]=\Sigma_{j=1}^{m}\left[A_{j}^{\prime}\right] \mid \forall p \exists \sigma_{p}\left[A_{j}^{\prime}\right]^{p}=\left[A_{\sigma_{p}(j)}\right]^{p} \forall 1 \leq j \leq m\right\} \\
& \stackrel{\simeq}{\longrightarrow} K_{0}\left(\mathrm{CSA}(k)^{\oplus}\right)^{+}
\end{aligned}
$$

where $p$ is a prime number and $\sigma_{p}$ a permutation of the set $\{1, \ldots, m\}$. Thanks to Lemma 4.26 below, the left-hand side of (4.24) may be replaced by the following semi-ring

$\mathbb{N}[\operatorname{Br}(k)] /\left\{\left[A^{\prime \prime}\right]+\left[A \otimes A^{\prime} \otimes A^{\prime \prime}\right]=\left[A \otimes A^{\prime \prime}\right]+\left[A \otimes A^{\prime \prime}\right] \mid\left(\operatorname{ind}(A), \operatorname{ind}\left(A^{\prime}\right)\right)=1\right\}$, 
where $A$ and $A^{\prime}$ are central simple $k$-algebras with coprime indexes. Therefore, by passing to group-completion, we obtain an induced (ring) isomorphism:

$$
\begin{aligned}
& \mathbb{Z}[\operatorname{Br}(k)] /\left\langle\left[A^{\prime \prime}\right]+\left[A \otimes A^{\prime} \otimes A^{\prime \prime}\right]-\left[A \otimes A^{\prime}\right]-\left[A \otimes A^{\prime \prime}\right] \mid\left(\operatorname{ind}(A), \operatorname{ind}\left(A^{\prime}\right)\right)=1\right\rangle \\
& \quad \simeq K_{0}\left(\mathrm{CSA}(k)^{\oplus}\right) .
\end{aligned}
$$

Finally, the proof follows now from the fact that the left-hand side of (4.25) agrees with the ring $R_{\mathrm{B}}(k)$.

Lemma 4.26 The following two sets of relations on the semi-ring $\mathbb{N}[\operatorname{Br}(k)]$ are equivalent:

$$
\left\{\sum_{j=1}^{m}\left[A_{j}\right]=\Sigma_{j=1}^{m}\left[A_{j}^{\prime}\right] \mid \forall p \exists \sigma_{p}\left[A_{j}^{\prime}\right]^{p}=\left[A_{\sigma_{p}(j)}\right]^{p} \forall 1 \leq j \leq m\right\}
$$

$$
\left\{\left[A^{\prime \prime}\right]+\left[A \otimes A^{\prime} \otimes A^{\prime \prime}\right]=\left[A \otimes A^{\prime \prime}\right]+\left[A \otimes A^{\prime \prime}\right] \mid\left(\operatorname{ind}(A), \operatorname{ind}\left(A^{\prime}\right)\right)=1\right\} .
$$

Proof Note first that the set (4.28) is contained in the set (4.27). Note also that since every permutation $\sigma_{p}$ can be written as a composition of transpositions, (4.27) is equivalent to the following set of relations

$$
\begin{aligned}
& \left\{\left[A_{1}\right]+\left[A_{2}\right]=\left[A_{1}^{\prime}\right]+\left[A_{2}^{\prime}\right] \mid\left[A_{1}^{\prime}\right]^{q}=\left[A_{2}\right]^{q},\left[A_{2}^{\prime}\right]^{q}=\left[A_{1}\right]^{q} \text { and }\left[A_{1}^{\prime}\right]^{p}\right. \\
& \left.\quad=\left[A_{1}\right]^{p},\left[A_{2}^{\prime}\right]^{p}=\left[A_{2}\right]^{p} \forall p \neq q\right\}
\end{aligned}
$$

where $q \neq p$ is a(ny) prime number. Therefore, it suffices to show that every relation in (4.29) is a particular case of a relation in (4.28). Recall from the Artin-Wedderburn theorem that $A_{1}$, resp. $A_{2}$, may be written as the matrix algebra of a unique central division $k$-algebra $D_{1}$, resp. $D_{2}$. Let us write $D_{1}=\otimes_{p} D_{1}^{p}$, resp. $D_{2}=\otimes_{p} D_{2}^{p}$, for the associated $p$-primary decomposition; consult [9, Prop. 4.5.16]. By construction, we have $\left[D_{1}^{p}\right]=\left[D_{1}\right]^{p}=\left[A_{1}\right]^{p}$, resp. $\left[D_{2}^{p}\right]=\left[D_{2}\right]^{p}=\left[A_{2}\right]^{p}$. Now, consider the following central simple $k$-algebras:

$$
A:=\left(D_{1}^{q}\right)^{\mathrm{op}} \otimes D_{2}^{q} A^{\prime}:=\left(\otimes_{p \neq q} D_{1}^{p}\right)^{\mathrm{op}} \otimes\left(\otimes_{p \neq q} D_{2}^{p}\right) A^{\prime \prime}:=A_{1} .
$$

Note that $\left(\operatorname{ind}(A)\right.$, ind $\left.\left(A^{\prime}\right)\right)=1$ and that for these choices the relation in (4.28) reduces to the relation:

$$
\left[A_{1}\right]+\left[D_{2}\right]=\left[\left(\otimes_{p \neq q} D_{1}^{p}\right) \otimes D_{2}^{q}\right]+\left[D_{1}^{q} \otimes\left(\otimes_{p \neq q} D_{2}^{p}\right)\right] .
$$

Making use of the equalities $\left[D_{2}\right]=\left[A_{2}\right],\left[\left(\otimes_{p \neq q} D_{1}^{p}\right) \otimes D_{2}^{q}\right]=\left[A_{1}^{\prime}\right]$ and $\left[D_{1}^{q} \otimes\right.$ $\left.\left(\otimes_{p \neq q} D_{2}^{p}\right)\right]=\left[A_{2}^{\prime}\right]$, we hence conclude that (4.30) agrees with the relation in (4.29). This finishes the proof.

Remark 4.31 (Characteristic zero) Note that in the above proof of Theorem 1.2 the assumption that $k$ is of characteristic zero is used solely in Bittner's presentation [3, Thm. 3.1] of the Grothendieck ring of varieties $K_{0} \operatorname{Var}(k)$; the remainder of the proof holds mutatis mutandis in arbitrary characteristic. 


\section{Properties of the Jacques Tits motivic measure}

Proposition 5.1 Let $\left\{{ }_{\gamma} \mathcal{F}_{j}\right\}_{1 \leq j \leq m}$ and $\left\{\gamma^{\prime} \mathcal{F}_{j}^{\prime}\right\}_{1 \leq j \leq m^{\prime}}$ be two families of twisted projective homogeneous varieties with Tits central simple k-algebras $\cup_{j=1}^{m}\left\{A_{\rho_{i j}}\right\}_{1 \leq i \leq n\left(\mathcal{F}_{j}\right)}$ and $\cup_{j=1}^{m^{\prime}}\left\{A_{\rho_{i_{j}}^{\prime}}^{\prime}\right\}_{1 \leq i \leq n}\left(\mathcal{F}_{j}^{\prime}\right)$.

(i) We have an isomorphism $U\left(\operatorname{perf}_{\mathrm{dg}}\left(\Pi_{j=1 \gamma}^{m} \mathcal{F}_{j}\right)\right) \simeq U\left(\operatorname{perf}_{\mathrm{dg}}\left(\Pi_{j=1 \gamma^{\prime}}^{m^{\prime}} \mathcal{F}_{j}^{\prime}\right)\right)$ in the category $\operatorname{NChow}(k)$ if and only if $\mu_{\mathrm{JT}}\left(\left[\Pi_{j=1 \gamma}^{m} \mathcal{F}_{j}\right]\right)=\mu_{\mathrm{JT}}\left(\left[\Pi_{j=1 \gamma^{\prime}}^{m^{\prime}} \mathcal{F}_{j}^{\prime}\right]\right)$ in $R_{\mathrm{B}}(k)$.

(ii) If $\mu_{\mathrm{JT}}\left(\left[\Pi_{j=1 \gamma}^{m} \mathcal{F}_{j}\right]\right)=\mu_{\mathrm{JT}}\left(\left[\Pi_{j=1 \gamma^{\prime}}^{m^{\prime}} \mathcal{F}_{j}^{\prime}\right]\right)$ in $R_{\mathrm{B}}(k)$, then $\left\langle\cup_{j=1}^{m}\left\{\left[A_{\rho_{i j}}\right]\right\}_{1 \leq i \leq n\left(\mathcal{F}_{j}\right)}\right\rangle$ $=\left\langle\cup_{j=1}^{m^{\prime}}\left\{\left[A_{\rho_{i_{j}}^{\prime}}^{\prime}\right]\right\}_{1 \leq i \leq n\left(\mathcal{F}_{j}^{\prime}\right)}\right\rangle$.

Proof Note first that an iterated application of Remark 3.2 leads to the following isomorphisms:

$$
\begin{aligned}
& U\left(\operatorname{perf}_{\mathrm{dg}}\left(\Pi_{j=1 \gamma}^{m} \mathcal{F}_{j}\right)\right) \simeq \otimes_{j=1}^{m} U\left(\operatorname{perf}_{\mathrm{dg}}\left(\gamma \mathcal{F}_{j}\right)\right) \simeq \otimes_{j=1}^{m} \oplus_{i=1}^{n\left(\mathcal{F}_{j}\right)} U\left(A_{\rho_{i j}}\right) \\
& U\left(\operatorname{perf}_{\mathrm{dg}}\left(\Pi_{j=1 \gamma^{\prime}}^{m^{\prime}} \mathcal{F}_{j}^{\prime}\right)\right) \simeq \otimes_{j=1}^{m^{\prime}} U\left(\operatorname{perf}_{\mathrm{dg}}\left(\gamma^{\prime} \mathcal{F}_{j}^{\prime}\right)\right) \simeq \otimes_{j=1}^{m^{\prime}} \oplus_{i=1}^{n\left(\mathcal{F}_{j}^{\prime}\right)} U\left(A_{\rho_{i_{j}}^{\prime}}^{\prime}\right) .
\end{aligned}
$$

Suppose that we have an isomorphism $U\left(\operatorname{perf}_{\mathrm{dg}}\left(\Pi_{j=1 \gamma}^{m} \mathcal{F}_{j}\right)\right) \simeq U\left(\operatorname{perf}_{\mathrm{dg}}\left(\Pi_{j=1 \gamma^{\prime}}^{m^{\prime}} \mathcal{F}_{j}^{\prime}\right)\right)$ in the category $\operatorname{NChow}(k)$. Since the noncommutative Chow motives (5.2)-(5.3) belong to the subcategory $\operatorname{CSA}(k)^{\oplus}$, it follows from the construction of the Tits motivic measure that $\mu_{\mathrm{JT}}\left(\left[\Pi_{j=1 \gamma}^{m} \mathcal{F}_{j}\right]\right)=\mu_{\mathrm{JT}}\left(\left[\Pi_{j=1 \gamma^{\prime}}^{m^{\prime}} \mathcal{F}_{j}^{\prime}\right]\right)$ in $R_{\mathrm{B}}(k)$. Conversely, if $\mu_{\mathrm{JT}}\left(\left[\Pi_{j=1 \gamma}^{m} \mathcal{F}_{j}\right]\right)=\mu_{\mathrm{JT}}\left(\left[\Pi_{j=1 \gamma^{\prime}}^{m^{\prime}} \mathcal{F}_{j}^{\prime}\right]\right)$ in $R_{\mathrm{B}}(k)$, then it follows from the construction of the Tits motivic measure that $\left[U\left(\operatorname{perf}_{\mathrm{dg}}\left(\Pi_{j=1 \gamma}^{m} \mathcal{F}_{j}\right)\right)\right]=\left[U\left(\operatorname{perf}_{\mathrm{dg}}\left(\Pi_{j=1 \gamma^{\prime}}^{m^{\prime}} \mathcal{F}_{j}^{\prime}\right)\right)\right]$ in $K_{0}(\operatorname{NChow}(k))$. By definition of $K_{0}(\operatorname{NChow}(k))$, this implies that there exists a noncommutative Chow motive $N M$ such that

$$
U\left(\operatorname{perf}_{\mathrm{dg}}\left(\Pi_{j=1 \gamma}^{m} \mathcal{F}_{j}\right)\right) \oplus N M \simeq U\left(\operatorname{perf}_{\mathrm{dg}}\left(\Pi_{j=1 \gamma^{\prime}}^{m^{\prime}} \mathcal{F}_{j}^{\prime}\right)\right) \oplus N M
$$

Since the noncommutative Chow motives (5.2-5.3) belong to the subcategory $\operatorname{CSA}(k)^{\oplus}$, we hence conclude from the cancellation Proposition 4.9 [applied to (5.4)] that $U\left(\operatorname{perf}_{\mathrm{dg}}\left(\Pi_{j=1 \gamma}^{m} \mathcal{F}_{j}\right)\right) \simeq U\left(\operatorname{perf}_{\mathrm{dg}}\left(\Pi_{j=1 \gamma^{\prime}}^{m^{\prime}} \mathcal{F}_{j}^{\prime}\right)\right)$ in the category NChow $(k)$. This proves item (i). Item (ii) follows now from item (i), from the fact that the noncommutative Chow motives (5.2-5.3) belong to the subcategory $\operatorname{CSA}(k)^{\oplus}$, and from Corollary 4.8 .

Lemma 5.5 The assignment $\left[{ }_{\gamma} \mathcal{F}\right] \mapsto n(\mathcal{F})$ gives rise to a motivic measure $\mu_{\rho}: K_{0} \operatorname{Var}(k)^{\mathrm{tw}} \rightarrow \mathbb{Z}$.

Proof The ring $R_{\mathrm{B}}(k)$ comes equipped with the augmentation $\Sigma_{j=1}^{m} n_{j}\left[A_{j}\right] \mapsto$ $\Sigma_{j=1}^{m} n_{j}$. By pre-composing this augmentation with the Tits motivic measure $\mu_{\mathrm{JT}}$, we hence obtain the motivic measure $\mu_{\rho}$. 


\section{Proof of Theorem 2.2}

Item (i). Recall first that $\operatorname{dim}(\operatorname{SB}(A))=\operatorname{deg}(A)-1$. Following $\S 2.1$, note that $\mu_{\rho}([\mathrm{SB}(A)])=\operatorname{deg}(A)$. Therefore, if $[\mathrm{SB}(A)]=\left[\mathrm{SB}\left(A^{\prime}\right)\right]$ in the Grothendieck ring of varieties $K_{0} \operatorname{Var}(k)$, we conclude that $\operatorname{deg}(A)=\operatorname{deg}\left(A^{\prime}\right)$. This is equivalent to the equality $\operatorname{dim}(\operatorname{SB}(A))=\operatorname{dim}\left(\operatorname{SB}\left(A^{\prime}\right)\right)$.

Item (ii). If $[\mathrm{SB}(A)]=\left[\mathrm{SB}\left(A^{\prime}\right)\right]$ in the Grothendieck ring of varieties $K_{0} \operatorname{Var}(k)$, then it follows from Proposition 5.1(ii) that $\langle[A]\rangle=\left\langle\left[A^{\prime}\right]\right\rangle$. In particular, we have $\operatorname{per}(A)=\operatorname{per}\left(A^{\prime}\right)$.

Item (iii). When $[A] \in{ }_{2} \operatorname{Br}(k)$, it follows from the equality $\langle[A]\rangle=\left\langle\left[A^{\prime}\right]\right\rangle$ (proved in item (ii)) that $[A]=\left[A^{\prime}\right]$ in the Brauer group $\operatorname{Br}(k)$. Using the fact that $\operatorname{deg}(A)=$ $\operatorname{deg}\left(A^{\prime}\right)$ (proved in item (i)), we hence conclude that $A \simeq A^{\prime}$. This implies that $\mathrm{SB}(A) \simeq \mathrm{SB}\left(A^{\prime}\right)$.

Item (iv). When $\operatorname{per}(A) \in\{3,4,5,6\}$, it follows from the equality $\langle[A]\rangle=\left\langle\left[A^{\prime}\right]\right\rangle$ (proved in item (ii)) that $[A]$ is equal to $\left[A^{\prime}\right]$, to $-\left[A^{\prime}\right]$, to $2\left[A^{\prime}\right]$, or to $-2\left[A^{\prime}\right]$. In the case where $[A]=\left[A^{\prime}\right]$, a proof similar to the one of item (iii) implies that $\mathrm{SB}(A) \simeq \mathrm{SB}\left(A^{\prime}\right)$. In the case where $[A]=-\left[A^{\prime}\right]$, the Amitsur conjecture holds thanks to the work of Roquette [23]. Hence, following item (iv') of Remark 2.7, we conclude that $\mathrm{SB}(A)$ and $\mathrm{SB}\left(A^{\prime}\right)$ are birational to each other. In the case where $[A]=2\left[A^{\prime}\right]$ (or $[A]=-2\left[A^{\prime}\right]$ ), the Amitsur conjecture holds thanks to the work of Tregub [31]. Hence, we conclude similarly that $\mathrm{SB}(A)$ and $\mathrm{SB}\left(A^{\prime}\right)$ are birational to each other.

Item (v). It is well known that if $\operatorname{deg}(A)=\operatorname{deg}\left(A^{\prime}\right)$ and if $\langle[A]\rangle=\left\langle\left[A^{\prime}\right]\right\rangle$, then the Severi-Brauer varieties $\mathrm{SB}(A)$ and $\mathrm{SB}\left(A^{\prime}\right)$ are stably birational to each other; consult, for example, [9, Rk. 5.4.3]. Consequently, the proof of item (v) follows from the above items (i) and (ii).

\section{Proof of Proposition 2.9}

Item (i). If $\left[C(a, b) \times C\left(a^{\prime}, b^{\prime}\right)\right]=\left[C\left(a^{\prime \prime}, b^{\prime \prime}\right) \times C\left(a^{\prime \prime \prime}, b^{\prime \prime \prime}\right)\right]$ in the Grothendieck ring of varieties $K_{0} \operatorname{Var}(k)$, then it follows from Proposition 5.1(ii) that $\left\langle[Q],\left[Q^{\prime}\right]\right\rangle=$ $\left\langle\left[Q^{\prime \prime}\right],\left[Q^{\prime \prime \prime}\right]\right\rangle$. Since $[Q],\left[Q^{\prime}\right],\left[Q^{\prime \prime}\right],\left[Q^{\prime \prime \prime}\right] \in{ }_{2} \operatorname{Br}(k)$, the latter equality implies that $[Q]$ (or $\left[Q^{\prime}\right]$ ) is equal to $\left[Q^{\prime \prime}\right]$ or to $\left[Q^{\prime \prime \prime}\right]$. Using the fact that $\operatorname{deg}(Q)=\operatorname{deg}\left(Q^{\prime}\right)=$ $\operatorname{deg}\left(Q^{\prime \prime}\right)=\operatorname{deg}\left(Q^{\prime \prime \prime}\right)=2$, we hence conclude that $Q$ (or $Q^{\prime}$ ) is isomorphic to $Q^{\prime \prime}$ or to $Q^{\prime \prime \prime}$. Equivalently, the conic $C(a, b)$ (or $\left.C\left(a^{\prime}, b^{\prime}\right)\right)$ is isomorphic to $C\left(a^{\prime \prime}, b^{\prime \prime}\right)$ or to $C\left(a^{\prime \prime \prime}, b^{\prime \prime \prime}\right)$.

Item (ii). When the quaternion $k$-algebras $Q$ and $Q^{\prime}$ are unlinked, i.e., when $Q \otimes Q^{\prime}$ is a division $k$-algebra, we have $\operatorname{ind}\left(Q \otimes Q^{\prime}\right)=4$. Consequently, using the fact that $\operatorname{ind}(Q)=\operatorname{ind}\left(Q^{\prime}\right)=\operatorname{ind}\left(Q^{\prime \prime}\right)=\operatorname{ind}\left(Q^{\prime \prime \prime}\right)=2$, we conclude from the equality $\left\langle[Q],\left[Q^{\prime}\right]\right\rangle=\left\langle\left[Q^{\prime \prime}\right],\left[Q^{\prime \prime \prime}\right]\right\rangle$ (proved in item (i)) that $[Q]=\left[Q^{\prime \prime}\right]$ and $\left[Q^{\prime}\right]=\left[Q^{\prime \prime \prime}\right]$ or that $[Q]=\left[Q^{\prime \prime \prime}\right]$ and $\left[Q^{\prime}\right]=\left[Q^{\prime \prime}\right]$. Since $\operatorname{deg}(Q)=\operatorname{deg}\left(Q^{\prime}\right)=\operatorname{deg}\left(Q^{\prime \prime}\right)=$ $\operatorname{deg}\left(Q^{\prime \prime \prime}\right)=2$, this implies that $Q \simeq Q^{\prime \prime}$ and $Q^{\prime} \simeq Q^{\prime \prime \prime}$ or that $Q \simeq Q^{\prime \prime \prime}$ and $Q^{\prime} \simeq Q^{\prime \prime}$. Equivalently, we have $C(a, b) \simeq C\left(a^{\prime \prime}, b^{\prime \prime}\right)$ and $C\left(a^{\prime}, b^{\prime}\right) \simeq C\left(a^{\prime \prime \prime}, b^{\prime \prime \prime}\right)$ 
or $C\left(a^{\prime}, b^{\prime}\right) \simeq C\left(a^{\prime \prime \prime}, b^{\prime \prime \prime}\right)$ and $C\left(a^{\prime}, b^{\prime}\right) \simeq C\left(a^{\prime \prime}, b^{\prime \prime}\right)$. In both cases we have an isomorphism $C(a, b) \times C\left(a^{\prime}, b^{\prime}\right) \simeq C\left(a^{\prime \prime}, b^{\prime \prime}\right) \times C\left(a^{\prime \prime \prime}, b^{\prime \prime \prime}\right)$.

\section{Proof of Theorem 2.13}

Item (i). Recall first that $\operatorname{dim}(\operatorname{Gr}(d ; A))=d \times(\operatorname{deg}(A)-d)$. Following $\S 2.3$, note that $\mu_{\rho}([\operatorname{Gr}(d ; A)])=\left(\begin{array}{c}\operatorname{deg}(A) \\ d\end{array}\right)$ because this is the number of Young diagrams inside the rectangle with $d$ lines and $\operatorname{deg}(A)-d$ columns. Hence, if $[\operatorname{Gr}(d ; A)]=\left[\operatorname{Gr}\left(d^{\prime} ; A^{\prime}\right)\right]$ in the Grothendieck ring of varieties $K_{0} \operatorname{Var}(k)$, we conclude that $\left(\begin{array}{c}\operatorname{deg}(A) \\ d\end{array}\right)=\left(\begin{array}{c}\operatorname{deg}\left(A^{\prime}\right) \\ d^{\prime}\end{array}\right)$. By definition of the binomial coefficients, this implies that $\operatorname{deg}(A)=\operatorname{deg}\left(A^{\prime}\right)$ and that $d=d^{\prime}$ or that $d^{\prime}=\operatorname{deg}(A)-d$. In both cases, we have $\operatorname{dim}(\operatorname{Gr}(d ; A))=$ $\operatorname{dim}\left(\operatorname{Gr}\left(d^{\prime} ; A^{\prime}\right)\right)$.

Item (ii). If $[\operatorname{Gr}(d ; A)]=\left[\operatorname{Gr}\left(d^{\prime} ; A^{\prime}\right)\right]$ in the Grothendieck ring of varieties $K_{0} \operatorname{Var}(k)$, then it follows from Proposition 5.1(ii) that $\langle[A]\rangle=\left\langle\left[A^{\prime}\right]\right\rangle$. In particular, we have $\operatorname{per}(A)=\operatorname{per}\left(A^{\prime}\right)$.

Item (iii). When $[A] \in{ }_{2} \operatorname{Br}(k)$, it follows from the equality $\langle[A]\rangle=\left\langle\left[A^{\prime}\right]\right\rangle$ (proved in item (ii)) that $[A]=\left[A^{\prime}\right]$ in the Brauer group $\operatorname{Br}(k)$. Using the fact that $\operatorname{deg}(A)=$ $\operatorname{deg}\left(A^{\prime}\right)$ (proved in item (i)), we hence conclude that $A \simeq A^{\prime}$. Since $d=d^{\prime}$ or $d=\operatorname{deg}(A)-d^{\prime}$, this implies that $\operatorname{Gr}(d ; A) \simeq \operatorname{Gr}\left(d^{\prime} ; A^{\prime}\right)$.

\section{Proof of Theorem 2.15}

Item (i). Recall first that $\operatorname{dim}\left(Q_{q}\right)=n-2$. Following $\S 2.4$, note that $\mu_{\rho}\left(Q_{q}\right)$ is equal to $n$ when $n$ is even or to $n-1$ when $n$ is odd. Hence, if $\left[Q_{q}\right]=\left[Q_{q^{\prime}}\right]$ in the Grothendieck ring of varieties $K_{0} \operatorname{Var}(k)$, we conclude that $n=n^{\prime}$ when $n$ is even or that $n-1=n^{\prime}-1$ when $n$ is odd. In both cases, we have $\operatorname{dim}\left(Q_{q}\right)=\operatorname{dim}\left(Q_{q^{\prime}}\right)$. This is equivalent to the equality $n=n^{\prime}$.

Item (ii). If $\left[Q_{q}\right]=\left[Q_{q^{\prime}}\right]$ in the Grothendieck ring of varieties $K_{0} \operatorname{Var}(k)$, then it follows from Proposition 5.1(ii) that $\left\langle\left[C_{0}^{+}(q)\right]\right\rangle=\left\langle\left[C_{0}^{+}\left(q^{\prime}\right)\right]\right\rangle$ when $n$ is even or $\left\langle\left[C_{0}(q)\right]\right\rangle=\left\langle\left[C_{0}\left(q^{\prime}\right)\right]\right\rangle$ when $n$ is odd. Since $\left[C_{0}^{+}(q)\right],\left[C_{0}(q)\right] \in{ }_{2} \operatorname{Br}(k)$, this implies that $\left[C_{0}^{+}(q)\right]=\left[C_{0}^{+}\left(q^{\prime}\right)\right]$ when $n$ is even or $\left[C_{0}(q)\right]=\left[C_{0}\left(q^{\prime}\right)\right]$ when $n$ is odd. Using the fact that $\operatorname{deg}\left(C_{0}^{+}(q)\right)=2^{\frac{n}{2}-1}, \operatorname{deg}\left(C_{0}(q)\right)=2^{\left\lfloor\frac{n}{2}\right\rfloor}$, and $n=n^{\prime}$ (proved in item (i)), we hence conclude that $C_{0}^{+}(q) \simeq C_{0}^{+}\left(q^{\prime}\right)$ when $n$ is even or $C_{0}(q) \simeq C_{0}\left(q^{\prime}\right)$ when $n$ is odd.

Item (iii). When $n=6$, the assignment $q \mapsto C_{0}^{+}(q)$ gives rise to a one-to-one correspondence between similarity classes of non-degenerate quadratic forms with trivial discriminant of dimension 6 and isomorphism classes of biquaternion algebras; consult [12, Cor. 15.33]. Consequently, the proof follows from the combination of item (ii) with the general fact that two quadratic forms $q$ and $q^{\prime}$ are similar if and only if the associated quadric hypersurfaces $Q_{q}$ and $Q_{q^{\prime}}$ are isomorphic.

Item (iv). When $I^{3}(k)=0$, we have the following classification result: if $n=n^{\prime}$ and $C_{0}^{+}(q) \simeq C_{0}^{+}\left(q^{\prime}\right)$ when $n$ is even or $C_{0}(q) \simeq C_{0}\left(q^{\prime}\right)$ when $n$ is odd, then the quadratic forms $q$ and $q^{\prime}$ are similar; consult [8, Thm. 3']. Consequently, the proof 
follows from the combination of items (i)-(ii) with the general fact that two quadratic forms $q$ and $q^{\prime}$ are similar if and only if the quadric hypersurfaces $Q_{q}$ and $Q_{q^{\prime}}$ are isomorphic.

\section{Proof of items (i)-(ii) of Theorem 2.19}

Item (i). Recall first that $\operatorname{dim}\left(\Pi_{j=1}^{m} Q_{q_{j}}\right)=\Sigma_{j=1}^{m} \operatorname{dim}\left(Q_{q_{j}}\right)=m \times(n-2)$. Following $\S 2.4$, note that $\mu_{\rho}\left(\left[\Pi_{j=1}^{m} Q_{q_{j}}\right]\right)=\Pi_{j=1}^{m} \mu_{\rho}\left(\left[Q_{q_{j}}\right]\right)$ is equal to $n^{m}$ when $n$ is even or to $(n-1)^{m}$ when $n$ is odd. Hence, if $\left[\Pi_{j=1}^{m} Q_{q_{j}}\right]=\left[\Pi_{j=1}^{m^{\prime}} Q_{q_{j}^{\prime}}\right]$ in the Grothendieck ring of varieties $K_{0} \operatorname{Var}(k)$, we conclude that $n^{m}=n^{m^{\prime}}$ when $n$ is even or that $(n-1)^{m}=$ $(n-1)^{m^{\prime}}$ when $n$ is odd. In both cases, we have $\operatorname{dim}\left(\Pi_{j=1}^{m} Q_{q_{j}}\right)=\operatorname{dim}\left(\Pi_{j=1}^{m^{\prime}} Q_{q_{j}^{\prime}}\right)$. This is equivalent to the equality $m=m^{\prime}$.

Item (ii). If $\left[\Pi_{j=1}^{m} Q_{q_{j}}\right]=\left[\Pi_{j=1}^{m^{\prime}} Q_{q_{j}^{\prime}}\right]$ in the Grothendieck ring of varieties $K_{0} \operatorname{Var}(k)$, then it follows from Proposition 5.1(ii) that $\left\langle\left\{\left[C_{0}^{+}\left(q_{j}\right)\right]\right\}_{1 \leq j \leq m}\right\rangle=$ $\left\langle\left\{\left[C_{0}^{+}\left(q_{j}^{\prime}\right)\right]\right\}_{1 \leq j \leq m^{\prime}}\right\rangle$ when $n$ is even or $\left\langle\left\{\left[C_{0}\left(q_{j}\right)\right]\right\}_{1 \leq j \leq m}\right\rangle=\left\langle\left\{\left[C_{0}\left(q_{j}^{\prime}\right)\right]\right\}_{1 \leq j \leq m^{\prime}}\right\rangle$ when $n$ is odd.

\section{Proof of items (iii)-(iv)-(iv') of Theorem 2.19}

We start with some auxiliar results of independent interest:

Proposition 11.1 Let $q$ and $q^{\prime}$ be two non-degenerate quadratic forms with trivial discriminant of dimension $n$. When $n=6$ or $I^{3}(k)=0$, the following conditions are equivalent:

(i) We have $Q_{q} \simeq Q_{q^{\prime}}$.

(ii) We have an isomorphism $U\left(\operatorname{perf}_{\mathrm{dg}}\left(Q_{q}\right)\right) \simeq U\left(\operatorname{perf}_{\mathrm{dg}}\left(Q_{q^{\prime}}\right)\right)$ in the category $\operatorname{NChow}(k)$.

(iii) We have $\left[C_{0}^{+}(q)\right]=\left[C_{0}^{+}\left(q^{\prime}\right)\right]$ when $n$ is even or $\left[C_{0}(q)\right]=\left[C_{0}\left(q^{\prime}\right)\right]$ when $n$ is odd.

Proof The implication (i) $\Rightarrow$ (ii) is clear. If we have an isomorphism $U\left(\operatorname{perf}_{\mathrm{dg}}\left(Q_{q}\right)\right) \simeq$ $U$ perf $_{\mathrm{dg}}\left(Q_{q^{\prime}}\right)$ in the category $\operatorname{NChow}(k)$, then it follows from Proposition 5.1 that $\left\langle\left[C_{0}^{+}(q)\right]\right\rangle=\left\langle\left[C_{0}^{+}\left(q^{\prime}\right)\right]\right\rangle$ when $n$ is even or $\left\langle\left[C_{0}(q)\right]\right\rangle=\left\langle\left[C_{0}\left(q^{\prime}\right)\right]\right\rangle$ when $n$ is odd. Using the fact that $\left[C_{0}^{+}(q)\right],\left[C_{0}(q)\right] \in{ }_{2} \mathrm{Br}(k)$, we hence conclude that $\left[C_{0}^{+}(q)\right]=\left[C_{0}^{+}\left(q^{\prime}\right)\right]$ when $n$ is even or $\left[C_{0}(q)\right]=\left[C_{0}\left(q^{\prime}\right)\right]$ when $n$ is odd. This proves the implication (ii) $\Rightarrow$ (iii). In what concerns the implication (iii) $\Rightarrow$ (i), recall that $\operatorname{deg}\left(C_{0}^{+}(q)\right)=\operatorname{deg}\left(C_{0}^{+}\left(q^{\prime}\right)\right)=2^{\frac{n}{2}-1}$ and that $\operatorname{deg}\left(C_{0}(q)\right)=\operatorname{deg}\left(C_{0}\left(q^{\prime}\right)\right)=2^{\left\lfloor\frac{n}{2}\right\rfloor}$. Therefore, if $\left[C_{0}^{+}(q)\right]=\left[C_{0}^{+}\left(q^{\prime}\right)\right]$ when $n$ is even or $\left[C_{0}(q)\right]=\left[C_{0}\left(q^{\prime}\right)\right]$ when $n$ is odd, we have $C_{0}^{+}(q) \simeq C_{0}^{+}\left(q^{\prime}\right)$ when $n$ is even or $C_{0}(q) \simeq C_{0}\left(q^{\prime}\right)$ when $n$ is odd. When $n=6$, we hence conclude from the one-to-one correspondence $q \mapsto C_{0}^{+}(q)$ between similarity classes of non-degenerate quadratic forms with trivial discriminant of dimension 6 and isomorphism classes of quaternion algebras (consult [12, Cor. 15.33]), that the quadratic forms $q$ and $q^{\prime}$ are similar or, equivalently, that $Q_{q} \simeq Q_{q^{\prime}}$. In the same 
vein, when $I^{3}(k)=0$, we conclude from the classification result [8, Thm. 3'] (which asserts that if $C_{0}^{+}(q) \simeq C_{0}^{+}\left(q^{\prime}\right)$ when $n$ is even or $C_{0}(q) \simeq C_{0}\left(q^{\prime}\right)$ when $n$ is odd, then the quadratic forms $q$ and $q^{\prime}$ are similar) that the quadratic forms $q$ and $q^{\prime}$ are similar or, equivalently, that $Q_{q} \simeq Q_{q^{\prime}}$.

The next result may be understood as the $\otimes$-analogue of Proposition 4.9.

Proposition 11.2 ( $\otimes$-cancellation) Let $\left\{A_{j}\right\}_{1 \leq j \leq m}$ and $\left\{A_{j}^{\prime}\right\}_{1 \leq j \leq m^{\prime}}$ be two families of central simple $k$-algebras and $q$ a non-degenerate quadratic form with trivial discriminant of dimension $n \geq 5$. If we have an isomorphism $\oplus_{j=1}^{m} U\left(A_{j}\right) \otimes U\left(\operatorname{perf}_{\mathrm{dg}}\left(Q_{q}\right)\right) \simeq$ $\oplus_{j=1}^{m^{\prime}} U\left(A_{j}^{\prime}\right) \otimes U\left(\operatorname{perf}_{\mathrm{dg}}\left(Q_{q}\right)\right)$ in the category $\operatorname{NChow}(k)$, then $m=m^{\prime}$ and $\oplus_{j=1}^{m} U\left(A_{j}\right) \simeq \oplus_{j=1}^{m} U\left(A_{j}^{\prime}\right)$.

Proof We prove first the case where $n \geq 6$ is even. Recall from [25, Example 3.8] that, since the central simple $k$-algebras $C_{0}^{+}(q)$ and $C_{0}^{-}(q)$ are isomorphic, we have the following computation:

$$
\begin{aligned}
& U\left(\operatorname{perf}_{\mathrm{dg}}\left(Q_{q}\right)\right) \simeq U(k)^{\oplus(n-2)} \oplus U\left(C_{0}^{+}(q)\right) \oplus U\left(C_{0}^{-}(q)\right) \\
& \simeq U(k)^{\oplus(n-2)} \oplus U\left(C_{0}^{+}(q)\right)^{\oplus 2} .
\end{aligned}
$$

Consequently, we obtain the following computation:

$$
\oplus_{j=1}^{m} U\left(A_{j}\right) \otimes U\left(\operatorname{perf}_{\mathrm{dg}}\left(Q_{q}\right)\right) \simeq \oplus_{j=1}^{m} U\left(A_{j}\right)^{\oplus(n-2)} \oplus \oplus_{j=1}^{m} U\left(A \otimes C_{0}^{+}(q)\right)^{\oplus 2}
$$

Making use of (11.3), the given isomorphism $\oplus_{j=1}^{m} U\left(A_{j}\right) \otimes U\left(\operatorname{perf}_{\mathrm{dg}}\left(Q_{q}\right)\right) \simeq$ $\oplus_{j=1}^{m^{\prime}} U\left(A_{j}^{\prime}\right) \otimes U\left(\operatorname{perf}_{\mathrm{dg}}\left(Q_{q}\right)\right)$ in the category $\operatorname{NChow}(k)$ may then be re-written as the following isomorphism

$$
\begin{aligned}
& \oplus_{j=1}^{m} U\left(A_{j}\right)^{\oplus(n-2)} \oplus \oplus_{j=1}^{m} U\left(A_{j} \otimes C_{0}^{+}(q)\right)^{\oplus 2} \\
& \simeq \oplus_{j=1}^{m^{\prime}} U\left(A_{j}^{\prime}\right)^{\oplus(n-2)} \oplus \oplus_{j=1}^{m^{\prime}} U\left(A_{j}^{\prime} \otimes C_{0}^{+}(q)\right)^{\oplus 2}
\end{aligned}
$$

in the category $\operatorname{CSA}(k)^{\oplus}$. Therefore, by applying Proposition 4.7 to the isomorphism (11.4), we conclude that $(n-2) m+2 m=(n-2) m^{\prime}+2 m^{\prime}$, which implies that $m=m^{\prime}$. In order to prove that $\oplus_{j=1}^{m} U\left(A_{j}\right) \simeq \oplus_{j=1}^{m} U\left(A_{j}^{\prime}\right)$, we will also make use of Proposition 4.7. Concretely, we need to show that for every prime number $p$ the following two sets of Brauer classes

$$
\left\{\left[A_{1}\right]^{p}, \ldots,\left[A_{m}\right]^{p}\right\} \quad\left\{\left[A_{1}^{\prime}\right]^{p}, \ldots,\left[A_{m}^{\prime}\right]^{p}\right\}
$$

are the same up to permutation. Recall that $\left[C_{0}^{+}(q)\right] \in{ }_{2} \operatorname{Br}(k)$. Therefore, when $p \neq 2$, the isomorphism (11.4) combined with Proposition 4.7 implies that the following two 
sets are the same up to permutation

$$
\{\underbrace{\left[A_{1}\right]^{p}, \ldots,\left[A_{m}\right]^{p}}_{(n-2)}, \underbrace{\left[A_{1}\right]^{p}, \ldots,\left[A_{m}\right]^{p}}_{2}\}\{\underbrace{\left\{\left[A_{1}^{\prime}\right]^{p}, \ldots,\left[A_{m}^{\prime}\right]^{p}\right.}_{(n-2)}, \underbrace{\left[A_{1}^{\prime}\right]^{p}, \ldots,\left[A_{m}^{\prime}\right]^{p}}_{2}\},
$$

where the numbers below the parenthesis denote the number of copies. Clearly, this implies that the above sets (11.5) are also the same up to permutation. When $p=2$, the isomorphism (11.4) combined with Proposition 4.7 implies that the following two sets are the same up to permutation:

$$
\begin{aligned}
& \{\underbrace{\left[A_{1}\right]^{2}, \ldots,\left[A_{m}\right]^{2}}_{(n-2)}, \underbrace{\left[A_{1} \otimes C_{0}^{+}(q)\right]^{2}, \ldots,\left[A_{m} \otimes C_{0}^{+}(q)\right]^{2}}_{2}\} \\
& \{\underbrace{\left[A_{1}^{\prime}\right]^{2}, \ldots,\left[A_{m}^{\prime}\right]^{2}}_{(n-2)}, \underbrace{\left[A_{1}^{\prime} \otimes C_{0}^{+}(q)\right]^{2}, \ldots,\left[A_{m}^{\prime} \otimes C_{0}^{+}(q)\right]^{2}}_{2}\} .
\end{aligned}
$$

Note that in the case where $\left[C_{0}^{+}(q)\right]=[k]$, this also implies that the sets $(11.5)$ are the same up to permutation. Let us then assume that $\left[C_{0}^{+}(q)\right] \neq[k]$. In this case, each one of the sets (11.6-11.7) is equipped with a non-trivial involution induced by tensoring with $C_{0}^{+}(q)$ (we are implicitly ignoring the number of copies of each Brauer class). In particular, we have $\left[A_{j} \otimes C_{0}^{+}(q)\right]^{2} \neq\left[A_{j}\right]^{2}$ for every $1 \leq j \leq m$ and if there exist integers $r$ and $s$ such that $\left[A_{r} \otimes C_{0}^{+}(q)\right]^{2}=\left[A_{s}\right]^{2}$, then $\left[A_{r}\right]^{2}=\left[A_{s} \otimes C_{0}^{+}(q)\right]^{2}$. Consequently, there exist disjoint subsets $\left\{j_{1}, \underline{j}_{1}, \ldots, j_{r}, \underline{j}_{r}\right\}$ and $\left\{i_{1}, \ldots, i_{s}\right\}$ of the set $\{1, \ldots, m\}$ and integers $n_{1}, \underline{n}_{1}, \ldots, n_{r}, \underline{n}_{r} \geq 1$ and $l_{1}, \ldots, l_{s} \geq 1$ such that (11.6) agrees with the following set of distinct Brauer classes:

$$
\cup_{t=1}^{r}\{\underbrace{\left\{\left[A_{j_{t}}\right]^{2}\right.}_{(n-2) n_{t}+2 \underline{n}_{t}}, \overbrace{\left[\hat{A}_{\underline{j}_{t}}\right]^{2}}^{(n-2) \underline{n}_{t}+2 n_{t}} \cup \cup_{t=1}^{s}\{\underbrace{\left[A_{i_{t}}\right]^{2}}_{(n-2) l_{t}}, \underbrace{\left[A_{i_{t}} \otimes C_{0}^{+}(q)\right]^{2}}_{2 l_{t}}\} .
$$

Similarly, there exist disjoint subsets $\left\{j_{1}^{\prime}, \underline{j}_{1}^{\prime}, \ldots, j_{r^{\prime}}^{\prime}, \underline{j}_{r^{\prime}}^{\prime}\right\}$ and $\left\{i_{1}^{\prime}, \ldots, i_{s^{\prime}}^{\prime}\right\}$ of the set $\{1, \ldots, m\}$ and integers $n_{1}^{\prime}, \underline{n}_{1}^{\prime}, \ldots, n_{r^{\prime}}^{\prime}, \underline{n}_{r^{\prime}}^{\prime} \geq 1$ and $l_{1}^{\prime}, \ldots, l_{s^{\prime}}^{\prime} \geq 1$ such that (11.7) agrees with the set of distinct Brauer classes:

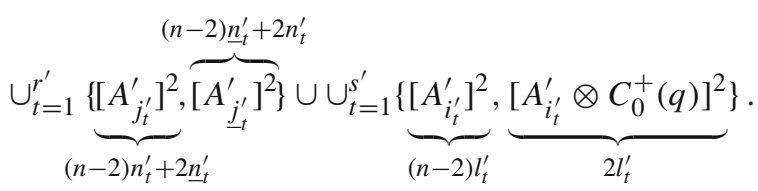

Note that, under these notations, the non-trivial involution on (11.8) (we are implicitly ignoring the number of copies of each Brauer class) interchanges $\left[A_{j_{t}}\right]^{2}$ with $\left[A_{\underline{j}_{t}}\right]^{2}$ and $\left[A_{i_{t}}\right]^{2}$ with $\left[A_{i_{t}} \otimes C_{0}^{+}(q)\right]^{2}$; similarly for the non-trivial involution on (11.9). Note also that, under these notations, the above sets (11.5) correspond to the following sets 
of Brauer classes (the Brauer classes in each one of the two sets are distinct):

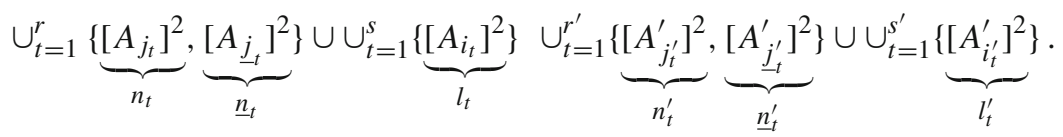

Now, recall that the above sets (11.8-11.9) are the same up to permutation. In other words, there exists a permutation with identifies the distinct Brauer classes of the set (11.8) with the distinct Brauer classes of the set (11.9). Therefore, making use of the non-trivial involutions on (11.8-11.9), of the precise number of copies of each Brauer class, and of the assumption that $n \geq 6$ is even, we hence conclude that the following sets of Brauer classes are the same up to permutation:

$$
\cup_{t=1}^{r} \underbrace{\left\{\left[A_{j_{t}}\right]^{2}\right.}_{(n-2) n_{t}+2 \underline{n}_{t}}, \overbrace{\left[A_{j_{t}}\right]^{2}}^{(n-2) \underline{n}_{t}+2 n_{t}} \cup_{t=1}^{r^{\prime}} \underbrace{\left\{\left[A_{j_{t}^{\prime}}^{\prime}\right]^{2}\right.}_{(n-2) n_{t}^{\prime}+2 \underline{n}_{t}^{\prime}}, \overbrace{\left[A_{\underline{j}_{t}^{\prime}}^{\prime}\right]^{2}}^{(n-2) \underline{n}_{t}^{\prime}+2 n_{t}^{\prime}} \text { resp. } \cup_{t=1}^{s}\{\underbrace{\left\{\left[A_{i_{t}}\right]^{2}\right.}_{(n-2) l_{t}}\} \quad \cup_{t=1}^{s^{\prime}}\{\underbrace{\left\{\left[A_{i_{t}^{\prime}}^{\prime}\right]^{2}\right\}}_{(n-2) l_{t}^{\prime}} .
$$

Making use once again of the non-trivial involution on the left-hand side of (11.11) and of the precise number of copies of each Brauer class, we observe that $r=r^{\prime}$ and that the sets $\left\{n_{1}, \underline{n}_{1}, \ldots, n_{r}, \underline{n}_{r}\right\}$ and $\left\{n_{1}^{\prime}, \underline{n}_{1}^{\prime}, \ldots, n_{r^{\prime}}^{\prime}, \underline{n}_{r^{\prime}}^{\prime}\right\}$ are the same up to permutation. In the same vein, we conclude from the right-hand side of (11.11) that $s=s^{\prime}$ and that the sets $\left\{l_{1}, \ldots, l_{s}\right\}$ and $\left\{l_{1}^{\prime}, \ldots, l_{s^{\prime}}^{\prime}\right\}$ are the same up to permutation. This implies that the following sets of Brauer classes are the same up to permutation:

$$
\left.\cup_{t=1}^{r}\{\underbrace{\left[A_{j_{t}}\right]^{2}}_{n_{t}}, \underbrace{\left[A_{\underline{j}_{t}}\right]^{2}}_{\underline{n}_{t}}\} \quad \cup_{t=1}^{r^{\prime}}\{\underbrace{\left[A_{j_{t}^{\prime}}^{\prime}\right]^{2}}_{n_{t}^{\prime}}, \underbrace{\left[A_{\underline{j}_{t}^{\prime}}^{]^{\prime}}\right.}_{\underline{n}_{t}^{\prime}}]^{2}\right\} \text { resp. } \cup_{t=1}^{s}\{\underbrace{\left[A_{i_{t}}\right]^{2}}_{l_{t}}\} \quad \cup_{t=1}^{s^{\prime}}\{\underbrace{\left\{\left[A_{i_{t}^{\prime}}^{\prime}\right]^{2}\right\}}_{l_{t}^{\prime}} .
$$

Consequently, by concatenating the permutations provided by (11.12), we hence obtain a permutation which identifies the left-hand side of (11.10) with the right-hand side of (11.10). In other words, the two sets in (11.10) are the same up to permutation. This proves the case where $n \geq 6$ is even. The proof of the case where $n \geq 5$ is odd is similar: simply replace the above isomorphism $U\left(\operatorname{perf}_{\mathrm{dg}}\left(Q_{q}\right)\right) \simeq U(k)^{\oplus(\bar{n}-2)} \oplus U\left(C_{0}^{+}(q)\right)^{\oplus 2}$ by the isomorphism $U$ (perf $\left._{\mathrm{dg}}\left(Q_{q}\right)\right) \simeq U(k)^{\oplus(n-2)} \oplus U\left(C_{0}(q)\right)$ and perform all the subsequent computations.

Lemma 11.13 Given integers $n \geq 5$ and $m, l \geq 0$, we have the implications (consult Notation 2.21):

$$
\begin{cases}\left(\Sigma_{\text {even }}^{1}(m, n, l)>\Sigma_{\text {even }}^{2}(m, n, l)\right) \Rightarrow\left(\Sigma_{\text {even }}^{1}(m-1, n, l)>\Sigma_{\text {even }}^{2}(m-1, n, l)\right) & \mathrm{n} \text { even } \\ \left(\Sigma_{\text {odd }}^{1}(m, n, l)>\Sigma_{\text {odd }}^{2}(m, n, l)\right) \Rightarrow\left(\Sigma_{\text {odd }}^{1}(m-1, n, l)>\Sigma_{\text {odd }}^{2}(m-1, n, l)\right) & \mathrm{n} \text { odd } .\end{cases}
$$


Proof Consider the following notations:

$$
\begin{aligned}
& \Sigma_{\text {even }}^{1,1}(m, n, l):=\Sigma_{r=0}^{\lfloor l / 2\rfloor}\left(\left(\begin{array}{c}
l \\
2 r
\end{array}\right) \times 2^{2 r+1} \times(n-2)^{m-(2 r+1)}\right) \\
& \Sigma_{\text {even }}^{1,2}(m, n, l):=\Sigma_{r=0}^{\lfloor l / 2\rfloor}\left(\left(\begin{array}{c}
l \\
2 r+1
\end{array}\right) \times 2^{m-l+(2 r+1)} \times(n-2)^{l-(2 r+1)}\right) \\
& \Sigma_{\text {odd }}^{1,1}(m, n, l):=\Sigma_{r=0}^{\lfloor l / 2\rfloor}\left(\left(\begin{array}{c}
l \\
2 r
\end{array}\right) \times(n-2)^{m-(2 r+1)}\right) \\
& \Sigma_{\text {odd }}^{1,2}(m, n, l):=\Sigma_{r=0}^{\lfloor l / 2\rfloor}\left(\left(\begin{array}{c}
l \\
2 r+1
\end{array}\right) \times(n-2)^{l-(2 r+1)}\right) .
\end{aligned}
$$

Note that $\Sigma_{\text {even }}^{1}(m, n, l)=\Sigma_{\text {even }}^{1,1}(m, n, l)+\Sigma_{\text {even }}^{1,2}(m, n, l)$ and $\Sigma_{\text {odd }}^{1}(m, n, l)=$ $\Sigma_{\text {odd }}^{1,1}(m, n, l)+\Sigma_{\text {odd }}^{1,2}(m, n, l)$. Note also that we have the following relations:

$$
\begin{aligned}
& \Sigma_{\text {even }}^{1,1}(m-1, n, l)=\frac{\Sigma_{\text {even }}^{1,1}(m, n, l)}{(n-2)} \\
& \Sigma_{\text {odd }}^{1,1}(m-1, n, l)=\frac{\Sigma_{\text {odd }}^{1,1}(m, n, l)}{(n-2)} \\
& \Sigma_{\text {even }}^{1,2}(m-1, n, l)=\frac{\Sigma_{\text {even }}^{1,2}(m, n, l)}{(n-2)} \\
& \Sigma_{\text {odd }}^{1,2}(m-1, n, l)=\Sigma_{\text {odd }}^{1,2}(m, n, l) \\
& \Sigma_{\text {even }}^{2}(m-1, n, l)=\frac{\Sigma_{\text {even }}^{2}(m, n, l)}{(n-2)} \\
& \Sigma_{\text {odd }}^{2}(m-1, n, l)=\frac{\Sigma_{\text {odd }}^{2}(m, n, l)}{(n-2)} .
\end{aligned}
$$

By combining them, we obtain the above implications (11.14).

We now have the ingredients necessary to prove items (iii)-(iv)-(iv') of Theorem 2.19. If $\left.\left.\left[\Pi_{j=1}^{m} Q_{q_{j}}\right)\right]=\left[\Pi_{j=1}^{m^{\prime}} Q_{q_{j}^{\prime}}\right)\right]$ in the Grothendieck ring of varieties $K_{0} \operatorname{Var}(k)$, then it follows from Theorem 2.19(i) that $m=m^{\prime}$. Moreover, we have $\left.\mu_{\mathrm{JT}}\left(\left[\Pi_{j=1}^{m} Q_{q_{j}}\right)\right]\right)=$ $\left.\mu_{\mathrm{JT}}\left(\left[\Pi_{j=1}^{m} Q_{q_{j}^{\prime}}\right)\right]\right)$ in $R_{\mathrm{B}}(k)$. Thanks to Proposition 5.1(i), the latter equality holds if and only if we have an isomorphism $U\left(\operatorname{perf}_{\mathrm{dg}}\left(\Pi_{j=1}^{m} Q_{q_{j}}\right)\right) \simeq U\left(\operatorname{perf}_{\mathrm{dg}}\left(\Pi_{j=1}^{m} Q_{q_{j}^{\prime}}\right)\right)$ in NChow $(k)$. Hence, the proof of items (iii)-(iv)-(iv') of Theorem 2.19 follows now from the following result:

Theorem 11.15 Let $\left\{q_{j}\right\}_{1 \leq j \leq m}$ and $\left\{q_{j}^{\prime}\right\}_{1 \leq j \leq m}$ be two families of non-degenerate quadratic forms with trivial discriminant of dimension $n \geq 5$. If we have an isomorphism $U\left(\operatorname{perf}_{\mathrm{dg}}\left(\Pi_{j=1}^{m} Q_{q_{j}}\right)\right) \simeq U\left(\operatorname{perf}_{\mathrm{dg}}\left(\Pi_{j=1}^{m} Q_{q_{j}^{\prime}}\right)\right)$ in the category $\operatorname{NChow}(k)$, then the following holds:

(iii) When $n=6$ and $m \leq 5$, we have $\Pi_{j=1}^{m} Q_{q_{j}} \simeq \Pi_{j=1}^{m} Q_{q_{j}^{\prime}}$.

(iv) When $I^{3}(k)=0$ and $m \leq 5$, we have $\Pi_{j=1}^{m} Q_{q_{j}} \simeq \Pi_{j=1}^{m} Q_{q_{j}^{\prime}}$. 
(iv') When $I^{3}(k)=0, m \geq 6$, and the following extra condition holds (consult Notation 2.21)

$$
\begin{cases}\Sigma_{\text {even }}^{1}(m, n, l)>\Sigma_{\text {even }}^{2}(m, n, l) \text { for all } 2 \leq l \leq m-3 & \text { n even } \\ \Sigma_{\text {odd }}^{1}(m, n, l)>\Sigma_{\text {odd }}^{2}(m, n, l) \text { for all } 2 \leq l \leq m-3 & \text { n odd }\end{cases}
$$

we also have $\Pi_{j=1}^{m} Q_{q_{j}} \simeq \Pi_{j=1}^{m} Q_{q_{j}^{\prime}}$.

Proof Recall from the proof of Proposition 11.2 that we have the following computation

$$
U\left(\operatorname{perf}_{\mathrm{dg}}\left(Q_{q_{j}}\right)\right) \simeq \begin{cases}U(k)^{\oplus(n-2)} \oplus U\left(C_{0}^{+}\left(q_{j}\right)\right)^{\oplus 2} & n \text { even } \\ U(k)^{\oplus(n-2)} \oplus U\left(C_{0}\left(q_{j}\right)\right) & n \text { odd }\end{cases}
$$

in the category $\operatorname{CSA}(k)^{\oplus}$. Following Remark 3.2, we have moreover the following isomorphisms:

$$
U\left(\operatorname{perf}_{\mathrm{dg}}\left(\Pi_{j=1}^{m} Q_{q_{j}}\right)\right) \simeq U\left(\otimes_{j=1}^{m} \operatorname{perf}_{\mathrm{dg}}\left(Q_{q_{j}}\right)\right) \simeq \otimes_{j=1}^{m} U\left(\operatorname{perf}_{\mathrm{dg}}\left(Q_{q_{j}}\right)\right)
$$

By combining (11.17-11.18), we hence obtain the following computation

$$
U\left(\operatorname{perf}_{\mathrm{dg}}\left(\Pi_{j=1}^{m} Q_{q_{j}}\right)\right) \simeq \begin{cases}\oplus_{S \subseteq\{1, \ldots, m\}} U\left(\otimes_{s \in S} C_{0}^{+}\left(q_{s}\right)\right)^{\oplus\left(2^{\#(S)} \times(n-2)^{m-\#(S)}\right)} & n \text { even } \\ \oplus_{S \subseteq\{1, \ldots, m\}} U\left(\otimes_{s \in S} C_{0}^{+}\left(q_{s}\right)\right)^{\oplus\left((n-2)^{m-\#(S)}\right)} & n \text { odd }\end{cases}
$$

where \#(S) stands for the cardinality of $S$ and $\otimes_{s \in \emptyset} C_{0}^{+}\left(q_{s}\right)=k$. Recall that $\left[C_{0}^{+}\left(q_{j}\right)\right],\left[C_{0}\left(q_{j}\right)\right] \in{ }_{2} \operatorname{Br}(k)$. Therefore, if we have an isomorphism $U\left(\operatorname{perf}_{\mathrm{dg}}\left(\Pi_{j=1}^{m}\right.\right.$ $\left.\left.Q_{q_{j}}\right)\right) \simeq U\left(\operatorname{perf}_{\mathrm{dg}}\left(\Pi_{j=1}^{m} Q_{q_{j}^{\prime}}\right)\right)$ in the category $\operatorname{NChow}(k)$, then it follows from Proposition 4.7 that the following sets of Brauer classes are the same up to permutation:

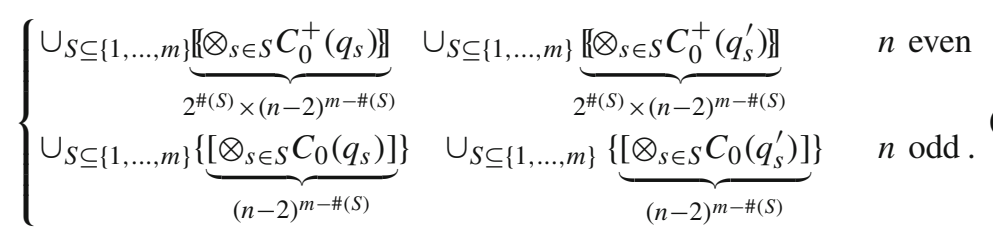

Moreover, Proposition 5.1(ii) yields the following equalities:

$$
\left\{\begin{array}{l}
\left\langle\left\{\left[C_{0}^{+}\left(q_{j}\right)\right]\right\}_{1 \leq j \leq m}\right\rangle=\left\langle\left\{\left[C_{0}^{+}\left(q_{j}^{\prime}\right)\right]\right\}_{1 \leq j \leq m}\right\rangle n \text { even } \\
\left\langle\left\{\left[C_{0}\left(q_{j}\right)\right]\right\}_{1 \leq j \leq m}\right\rangle=\left\langle\left\{\left[C_{0}\left(q_{j}^{\prime}\right)\right]\right\}_{1 \leq j \leq m}\right\rangle n \text { odd } .
\end{array}\right.
$$


Note that since $\left[C_{0}^{+}\left(q_{j}\right)\right],\left[C_{0}\left(q_{j}\right)\right] \in{ }_{2} \mathrm{Br}(k),(11.21)$ is a (finite-dimensional) $\mathbb{F}_{2}$ linear subspace of ${ }_{2} \mathrm{Br}(k)$.

Notation 11.22 Let us write $d$ for the dimension of the $\mathbb{F}_{2}$-vector space (11.21).

Item (iii). We will prove item (iii) by induction on $m \geq 1$. Note first that in the particular case where $m=1$, the proof follows from Proposition 11.1. Let us assume that item (iii) holds for $m-1$, with $m \in\{2,3,4,5\}$. Making use of (11.18), we have the following isomorphism

$$
\begin{aligned}
& U\left(\operatorname{perf}_{\mathrm{dg}}\left(\Pi_{j=1}^{m-1} Q_{q_{j}}\right)\right) \otimes U\left(\operatorname{perf}_{\mathrm{dg}}\left(Q_{q_{m}}\right)\right) \\
& \simeq U\left(\operatorname{perf}_{\mathrm{dg}}\left(\Pi_{j=1}^{m-1} Q_{q_{j}^{\prime}}\right)\right) \otimes U\left(\operatorname{perf}_{\mathrm{dg}}\left(Q_{q_{m}^{\prime}}\right)\right)
\end{aligned}
$$

in the category $\operatorname{NChow}(k)$. On the one hand, when $m \in\{2,3,4\}$, we have $d \in$ $\{0,1,2, m-1, m\}$. On the other hand, $d \in\{0,1,2,3,4,5\}$ when $m=5$. Moreover, when $d=3$, we have the following inequality:

$$
\Sigma_{\text {even }}^{1}(5,6,2)=2^{9}+2^{7}+2^{7}=768>576=2^{8}+2^{8}+2^{6}=\Sigma_{\text {even }}^{2}(5,6,2) .
$$

Therefore, thanks to Lemma 11.25 below, there exist integers $r$ and $s$ such that $Q_{q_{r}} \simeq Q_{q_{s}^{\prime}}$. Without loss of generality, we can assume that $Q_{q_{m}} \simeq Q_{q_{m}^{\prime}}$. By applying the $\otimes$-cancellation Proposition 11.2 to (11.23) (with $Q_{q}$ equal to $Q_{q_{m}} \simeq Q_{q_{m}^{\prime}}$ ), we hence obtain an isomorphism $U\left(\operatorname{perf}_{\mathrm{dg}}\left(\Pi_{j=1}^{m-1} Q_{q_{j}}\right)\right) \simeq U\left(\operatorname{perf}_{\mathrm{dg}}\left(\Pi_{j=1}^{m-1} Q_{q_{j}^{\prime}}\right)\right)$ in the category $\operatorname{NChow}(k)$. Using the assumption that item (iii) holds for $m-1$, we therefore conclude that $\Pi_{j=1}^{m-1} Q_{q_{j}} \simeq \Pi_{j=1}^{m-1} Q_{q_{j}^{\prime}}$. Consequently, the searched isomorphism $\Pi_{j=1}^{m} Q_{q_{j}} \simeq \Pi_{j=1}^{m} Q_{q_{j}^{\prime}}$ follows now from the combination of $\Pi_{j=1}^{m-1} Q_{q_{j}} \simeq \Pi_{j=1}^{m-1} Q_{q_{j}^{\prime}}$ with $Q_{q_{m}} \simeq Q_{q_{m}^{\prime}}$.

Item (iv). The proof is similar to the the proof of item (iii): simply replace the condition $n=6$ by the condition $I^{3}(k)=0$ and the computations of $\Sigma_{\text {even }}^{1}(5,6,2)$ and $\Sigma_{\text {even }}^{2}(5,6,2)$ by the following computations:

$$
\left\{\begin{array}{l}
\Sigma_{\text {even }}^{1}(5, n, 2)=2 \times(n-2)^{4}+2^{5} \times(n-2)+2^{3} \times(n-2)^{2} \\
\Sigma_{\text {odd }}^{1}(5, n, 2)=(n-2)^{4}+2 \times(n-2)+(n-2)^{2} \\
\Sigma_{\text {even }}^{2}(5, n, 2)=2^{2} \times(n-2)^{3}+2^{2} \times(n-2)^{3}+2^{4} \times(n-2) \\
\Sigma_{\text {odd }}^{2}(5, n, 2)=(n-2)^{3}+2 \times(n-2)^{3}+(n-2) .
\end{array}\right.
$$


A simple verification shows that $\Sigma_{\text {even }}^{1}(5, n, 2)>\Sigma_{\text {even }}^{2}(5, n, 2)$ and $\Sigma_{\text {odd }}^{1}(5, n, 2)>$ $\Sigma_{\text {odd }}^{2}(5, n, 2)$ when $n \geq 5$.

Item (iv'). We will prove item (iv') by induction on $m \geq 6$. Using (11.18), we have the isomorphism

$$
\begin{aligned}
& U\left(\operatorname{perf}_{\mathrm{dg}}\left(\Pi_{j=1}^{m-1} Q_{q_{j}}\right)\right) \otimes U\left(\operatorname{perf}_{\mathrm{dg}}\left(Q_{q_{m}}\right)\right) \\
& \simeq U\left(\operatorname{perf}_{\mathrm{dg}}\left(\Pi_{j=1}^{m-1} Q_{q_{j}^{\prime}}\right)\right) \otimes U\left(\operatorname{perf}_{\mathrm{dg}}\left(Q_{q_{m}^{\prime}}\right)\right)
\end{aligned}
$$

in the category $\operatorname{NChow}(k)$. Let us assume that item (iv') holds for $m-1$, with $m \geq 6$ (in the case where $m=6$, item (iv) holds). By definition, $d \in\{0,1,2, \ldots, m-1, m\}$. Moreover, when $d \in\{3, \ldots, m-2\}$, the extra condition (11.16) (with $l=$ $m-d)$ implies that $\Sigma_{\text {even }}^{1}(m, n, m-d)>\Sigma_{\text {even }}^{2}(m, n, m-d)$ when $n$ is even or $\Sigma_{\text {odd }}^{1}(m, n, m-d)>\Sigma_{\text {odd }}^{2}(m, n, m-d)$ when $n$ is odd. Therefore, thanks to Lemma 11.25 below, there exist integers $r$ and $s$ such that $Q_{q_{r}} \simeq Q_{q_{s}^{\prime}}$. Without loss of generality, we can assume that $Q_{q_{m}} \simeq Q_{q_{m}^{\prime}}$. By applying the $\otimes$-cancellation Proposition 11.2 to the isomorphism (11.24) (with $Q_{q}$ equal to $Q_{q_{m}} \simeq Q_{q_{m}^{\prime}}$ ), we hence obtain an isomorphism $U\left(\operatorname{perf}_{\mathrm{dg}}\left(\Pi_{j=1}^{m-1} Q_{q_{j}}\right)\right) \simeq U\left(\operatorname{perf}_{\mathrm{dg}}\left(\Pi_{j=1}^{m-1} Q_{q_{j}^{\prime}}\right)\right)$ in the category $\operatorname{NChow}(k)$. Now, note that Lemma 11.13 implies that if the extra condition (11.16) holds for $m$, then it also holds for $m-1$. Using the assumption that item (iv') holds for $m-1$ (in the case where $m=6$, item (iv) holds), we therefore conclude that $\Pi_{j=1}^{m-1} Q_{q_{j}} \simeq \Pi_{j=1}^{m-1} Q_{q_{j}^{\prime}}$. Consequently, the searched isomorphism $\Pi_{j=1}^{m} Q_{q_{j}} \simeq \Pi_{j=1}^{m} Q_{q_{j}^{\prime}}$ follows now from the combination of $\Pi_{j=1}^{m-1} Q_{q_{j}} \simeq \Pi_{j=1}^{m-1} Q_{q_{j}^{\prime}}$ with $Q_{q_{m}} \simeq Q_{q_{m}^{\prime}}$.

Lemma 11.25 Assume that $n=6$ or that $n \geq 5$ and $I^{3}(k)=0$.

(i) If $d \in\{0,1,2, m-1, m\}$ (consult Notation 11.22), then there exist integers $r$ and s such that $Q_{q_{r}} \simeq Q_{q_{s}^{\prime}}$.

(i') If $d \in\{3, \ldots, m-2\}$ and the following extra condition holds (consult Notation 2.21)

$$
\begin{cases}\Sigma_{\text {even }}^{1}(m, n, m-d)>\Sigma_{\text {even }}^{2}(m, n, m-d) & \mathrm{n} \text { even } \\ \Sigma_{\text {odd }}^{1}(m, n, m-d)>\Sigma_{\text {odd }}^{2}(m, n, m-d) & \mathrm{n} \text { odd }\end{cases}
$$

then there also exist integers $r$ and s such that $Q_{q_{r}} \simeq Q_{q_{s}^{\prime}}$.

Proof In the case where $d \in\{0,1,2\}$, it follows from the equalities (11.21) that there exist integers $r$ and $s$ such that $\left[C_{0}^{+}\left(q_{r}\right)\right]=\left[C_{0}^{+}\left(q_{s}^{\prime}\right)\right]$ when $n$ is even or $\left[C_{0}\left(q_{r}\right)\right]=\left[C_{0}\left(q_{s}^{\prime}\right)\right]$ when $n$ is odd. Making use of Proposition 11.1, we hence conclude that $Q_{q_{r}} \simeq Q_{q_{s}^{\prime}}$. In the case where $d=m$, it follows from the precise number of copies of each Brauer class in (11.20) that there exists a permutation $\sigma$ of the set $\{1, \ldots, m\}$ such that $\left[C_{0}^{+}\left(q_{j}^{\prime}\right)\right]=\left[C_{0}^{+}\left(q_{\sigma(j)}\right)\right]$ when $n$ is even or $\left[C_{0}\left(q_{j}^{\prime}\right)\right]=\left[C_{0}\left(q_{\sigma(j)}\right)\right]$ when $n$ is odd. In particular, there exist integers $r$ and $s$ such that $\left[C_{0}^{+}\left(q_{r}\right)\right]=\left[C_{0}^{+}\left(q_{s}^{\prime}\right)\right]$ when $n$ is even or $\left[C_{0}\left(q_{r}\right)\right]=\left[C_{0}\left(q_{s}^{\prime}\right)\right]$ when $n$ 
is odd. Making use of Proposition 11.1, we hence conclude that $Q_{q_{r}} \simeq Q_{q_{s}^{\prime}}$. In the case where $d=m-1$, let us suppose by absurd that $Q_{q_{r}} \nsucceq Q_{q_{s}^{\prime}}$ for every $1 \leq r, s \leq m$. Thanks to Proposition 11.1, this is equivalent to the condition that $\left[C_{0}^{+}\left(q_{r}\right)\right] \neq\left[C_{0}^{+}\left(q_{s}^{\prime}\right)\right]$ for every $1 \leq r, s \leq m$ when $n$ is even or $\left[C_{0}\left(q_{r}\right)\right] \neq\left[C_{0}\left(q_{s}^{\prime}\right)\right]$ for every $1 \leq r, s \leq m$ when $n$ is odd. Without loss of generality, we can assume that the $m-1$ vectors $\left\{\left[C_{0}^{+}\left(q_{j}\right)\right]\right\}_{1 \leq j \leq m-1}$, resp. $\left\{\left[C_{0}^{+}\left(q_{j}^{\prime}\right)\right]\right\}_{1 \leq j \leq m-1}$, of the $\mathbb{F}_{2-}$ vector space $\left\langle\left\{\left[C_{0}^{+}\left(q_{j}\right)\right]\right\}_{1 \leq j \leq m}\right\rangle$, resp. $\left\langle\left\{\left[C_{0}^{+}\left(q_{j}^{\prime}\right)\right]\right\}_{1 \leq j \leq m}\right\rangle$, are linearly independent when $n$ is even or that the $m-1$ vectors $\left\{\left[C_{0}\left(q_{j}\right)\right]\right\}_{1 \leq j \leq m-1},\left\{\left[C_{0}\left(q_{j}^{\prime}\right)\right]\right\}_{1 \leq j \leq m-1}$, of the $\mathbb{F}_{2}$-vector space $\left\langle\left\{\left[C_{0}\left(q_{j}\right)\right]\right\}_{1 \leq j \leq m}\right\rangle$, resp. $\left\langle\left\{\left[C_{0}\left(q_{j}^{\prime}\right)\right]\right\}_{1 \leq j \leq m}\right\rangle$, are linearly independent when $n$ is odd. On the one hand, this assumption implies that the lowest possible number of copies of the Brauer class $\left[C_{0}^{+}\left(q_{1}\right)\right]$, resp. $\left[C_{0}\left(q_{1}\right)\right]$, on the lefthand side of (11.20) is attained when $\left[C_{0}^{+}\left(q_{m}\right)\right]=\left[C_{0}^{+}\left(q_{2}\right) \otimes \ldots \otimes C_{0}^{+}\left(q_{m-1}\right)\right]$, resp. $\left[C_{0}\left(q_{m}\right)\right]=\left[C_{0}\left(q_{2}\right) \otimes \ldots \otimes C_{0}\left(q_{m-1}\right)\right]$. A simple computation shows that the corresponding number of copies is equal to $\Sigma_{\text {even }}^{1}(m, n, 1)$ when $n$ is even or $\Sigma_{\text {odd }}^{1}(m, n, 1)$ when $n$ is odd. On the other hand, the above assumption implies that the highest possible number of copies of the Brauer class $\left[C_{0}^{+}\left(q_{1}\right)\right]$, resp. $\left[C_{0}\left(q_{1}\right)\right]$, on the right-hand side of (11.20) is obtained when $\left[C_{0}^{+}\left(q_{1}\right)\right]=\left[C_{0}^{+}\left(q_{t}^{\prime}\right) \otimes C_{0}^{+}\left(q_{u}^{\prime}\right)\right]$, resp. $\left[C_{0}\left(q_{1}\right)\right]=\left[C_{0}\left(q_{t}^{\prime}\right) \otimes C_{0}\left(q_{u}^{\prime}\right)\right]$, for integers $1 \leq t \neq u \leq m-1$ and when $\left[C_{0}^{+}\left(q_{m}^{\prime}\right)\right]=\left[C_{0}^{+}\left(q_{v}^{\prime}\right)\right]$, resp. $\left[C_{0}\left(q_{m}^{\prime}\right)\right]=\left[C_{0}\left(q_{v}^{\prime}\right)\right]$, for an integer $1 \leq v \leq m-1$ (recall that we are supposing by absurd that $\left[C_{0}^{+}\left(q_{r}\right)\right] \neq\left[C_{0}^{+}\left(q_{s}^{\prime}\right)\right]$ for every $1 \leq r, s \leq m$ when $n$ is even or $\left[C_{0}\left(q_{r}\right)\right] \neq\left[C_{0}\left(q_{s}^{\prime}\right)\right]$ for every $1 \leq r, s \leq m$ when $n$ is odd). A simple computation shows that the corresponding number of copies is equal to $\Sigma_{\text {even }}^{1}(m, n, 1)$ when $n$ is even or $\Sigma_{\text {odd }}^{2}(m, n, 1)$ when $n$ is odd. Now, note that the following inequalities

$$
\begin{aligned}
\Sigma_{\text {even }}^{1}(m, n, 1) & =2 \times(n-2)^{m-1}+2^{m}>4 \times(n-2)^{m-2}+2 \times(n-2)^{m-2}=\Sigma_{\text {even }}^{2}(m, n, 1) \\
\Sigma_{\text {odd }}^{1}(m, n, 1) & =(n-2)^{m-1}+1>(n-2)^{m-2}+(n-2)^{m-2}=\Sigma_{\text {odd }}^{2}(m, n, 1)
\end{aligned}
$$

lead to a contradiction with the fact that the above two sets (11.20) are the same up to permutation. Consequently, there exist integers $r$ and $s$ such that $\left[C_{0}^{+}\left(q_{r}\right)\right]=\left[C_{0}^{+}\left(q_{s}^{\prime}\right)\right]$ when $n$ is even or $\left[C_{0}\left(q_{r}\right)\right]=\left[C_{0}\left(q_{s}^{\prime}\right)\right]$ when $n$ is odd. Making use of Proposition 11.1, we hence conclude that $Q_{q_{r}} \simeq Q_{q_{s}^{\prime}}$. This proves item (i).

We now prove item (i'). Let us suppose by absurd that $Q_{q_{r}} \nsucceq Q_{q_{s}^{\prime}}$ for every $1 \leq r, s \leq m$. Thanks to Proposition 11.1, this is equivalent to the condition that $\left[C_{0}^{+}\left(q_{r}\right)\right] \neq\left[C_{0}^{+}\left(q_{s}^{\prime}\right)\right]$ for every $1 \leq r, s \leq m$ when $n$ is even or $\left[C_{0}\left(q_{r}\right)\right] \neq\left[C_{0}\left(q_{s}^{\prime}\right)\right]$ for every $1 \leq r, s \leq m$ when $n$ is odd. Since $d \in\{3, \ldots, m-2\}$, we can assume, without loss of generality, that the $d$ vectors $\left\{\left[C_{0}^{+}\left(q_{j}\right)\right]\right\}_{1 \leq j \leq d}$, resp. $\left\{\left[C_{0}^{+}\left(q_{j}^{\prime}\right)\right]\right\}_{1 \leq j \leq d}$, of the $\mathbb{F}_{2}$-vector space $\left\langle\left\{\left[C_{0}^{+}\left(q_{j}\right)\right]\right\}_{1 \leq j \leq m}\right\rangle$, resp. $\left\langle\left\{\left[C_{0}^{+}\left(q_{j}^{\prime}\right)\right]\right\}_{1 \leq j \leq m}\right\rangle$, are linearly independent when $n$ is even or that the $d$ vectors $\left\{\left[C_{0}\left(q_{j}\right)\right]\right\}_{1 \leq j \leq d}$, resp. $\left\{\left[C_{0}\left(q_{j}^{\prime}\right)\right]\right\}_{1 \leq j \leq d}$, of the $\mathbb{F}_{2}$-vector space $\left\langle\left\{\left[C_{0}\left(q_{j}\right)\right]\right\}_{1 \leq j \leq m}\right\rangle$, resp. $\left\langle\left\{\left[C_{0}\left(q_{j}^{\prime}\right)\right]\right\}_{1 \leq j \leq m}\right\rangle$, are linearly independent when $n$ is odd. On the one hand, this assumption implies that the lowest possible number of copies of the Brauer class $\left[C_{0}^{+}\left(q_{1}\right)\right]$, resp. $\left[C_{0}\left(q_{1}\right)\right]$, on the lefthand side of (11.20) is attained when $\left[C_{0}^{+}\left(q_{d+1}\right)\right]=\cdots=\left[C_{0}^{+}\left(q_{m}\right)\right]=\left[C_{0}^{+}\left(q_{2}\right) \otimes\right.$ $\left.\cdots \otimes C_{0}^{+}\left(q_{m-1}\right)\right]$, resp. $\left[C_{0}\left(q_{d+1}\right)\right]=\cdots=\left[C_{0}\left(q_{m}\right)\right]=\left[C_{0}\left(q_{2}\right) \otimes \cdots \otimes C_{0}\left(q_{m-1}\right)\right]$. A simple computation shows that the corresponding number of copies is equal to 
$\Sigma_{\text {even }}^{1}(m, n, m-d)$ when $n$ is even or $\Sigma_{\text {odd }}^{1}(m, n, m-d)$ when $n$ is odd. On the other hand, the above assumption implies that the highest possible number of copies of Brauer class $\left[C_{0}^{+}\left(q_{1}\right)\right]$, resp. $\left[C_{0}\left(q_{1}\right)\right]$, on the right-hand side of $(11.20)$ is attained when $\left[C_{0}^{+}\left(q_{1}\right)\right]=\left[C_{0}^{+}\left(q_{t}^{\prime}\right) \otimes C_{0}^{+}\left(q_{u}^{\prime}\right)\right]$, resp. $\left[C_{0}\left(q_{1}\right)\right]=\left[C_{0}\left(q_{t}^{\prime}\right) \otimes C_{0}\left(q_{u}^{\prime}\right)\right]$, for integers $1 \leq t \neq u \leq d$ and when $\left[C_{0}^{+}\left(q_{d+1}^{\prime}\right)\right]=\cdots=\left[C_{0}^{+}\left(q_{m}^{\prime}\right)\right]=\left[C_{0}^{+}\left(q_{v}^{\prime}\right)\right]$, resp. $\left[C_{0}\left(q_{d+1}^{\prime}\right)\right]=\cdots=\left[C_{0}\left(q_{m}^{\prime}\right)\right]=\left[C_{0}\left(q_{v}^{\prime}\right)\right]$, for an integer $1 \leq v \leq d$. A simple computation shows that the corresponding number of copies is equal to $\Sigma_{\text {even }}^{2}(m, n, m-d)$ when $n$ is even or $\Sigma_{\text {odd }}^{2}(m, n, m-d)$ when $n$ is odd. Thanks to the inequalities (11.26), we hence obtain a contradiction with the fact that the above two sets (11.20) are the same up to permutation. Consequently, there exist integers $r$ and $s$ such that $\left[C_{0}^{+}\left(q_{r}\right)\right]=\left[C_{0}^{+}\left(q_{s}^{\prime}\right)\right]$ when $n$ is even or $\left[C_{0}\left(q_{r}\right)\right]=\left[C_{0}\left(q_{s}^{\prime}\right)\right]$ when $n$ is odd. Making use of Proposition 11.1, we hence conclude that $Q_{q_{r}} \simeq Q_{q_{s}^{\prime}}$.

\section{Proof of Theorem 2.23}

Item (i). Recall first that $\operatorname{dim}(\operatorname{Iv}(A, *))=\operatorname{deg}(A)$. Following $\$ 2.6$, note that $\mu_{\rho}(\operatorname{Iv}(A, *))=\operatorname{deg}(A)$. Hence, if $[\operatorname{Iv}(A, *)]=\left[\operatorname{Iv}\left(A^{\prime}, *^{\prime}\right)\right]$ in $K_{0} \operatorname{Var}(k)$, we conclude that $\operatorname{dim}(\operatorname{Iv}(A, *))=\operatorname{dim}\left(\operatorname{Iv}\left(A^{\prime}, *^{\prime}\right)\right)$. This is equivalent to the equality $\operatorname{deg}(A)=\operatorname{deg}\left(A^{\prime}\right)$.

Item (ii). If $[\operatorname{Iv}(A, *)]=\left[\operatorname{Iv}\left(A^{\prime}, *^{\prime}\right)\right]$ in $K_{0} \operatorname{Var}(k)$, then it follows from Proposition 5.1(ii) that

$$
\left\langle[A],\left[C_{0}^{+}(A, *)\right],\left[C_{0}^{-}(A, *)\right]\right\rangle=\left\langle\left[A^{\prime}\right],\left[C_{0}^{+}\left(A^{\prime}, *^{\prime}\right)\right],\left[C_{0}^{-}\left(A^{\prime}, *^{\prime}\right)\right]\right\rangle .
$$

When $\operatorname{deg}(A) \equiv 2(\bmod 4)$, we have the following relations in the Brauer group:

$$
2\left[C_{0}^{+}(A, *)\right]=[A] 3\left[C_{0}^{+}(A, *)\right]=\left[C_{0}^{-}(A, *)\right] \quad 4\left[C_{0}^{+}(A, *)\right]=[k] .
$$

Note that (12.1-12.2) imply that $\left[C_{0}^{+}(A, *)\right]=\left[C_{0}^{+}\left(A^{\prime}, *^{\prime}\right)\right]$ and $\left[C_{0}^{-}(A, *)\right]=$ $\left[C_{0}^{-}\left(A^{\prime}, *^{\prime}\right)\right]$ or that $\left[C_{0}^{+}(A, *)\right]=\left[C_{0}^{-}\left(A^{\prime}, *^{\prime}\right)\right]$ and $\left[C_{0}^{-}(A, *)\right]=\left[C_{0}^{+}\left(A^{\prime}, *^{\prime}\right)\right]$. Using the fact that $\operatorname{deg}\left(C_{0}^{+}(A, *)\right)=\operatorname{deg}\left(C_{0}^{-}(A, *)\right)=2^{\frac{\operatorname{deg}(A)}{2}-1}$ and that $\operatorname{deg}(A)=$ $\operatorname{deg}\left(A^{\prime}\right)$ (proved in item (i)), we hence conclude that $C_{0}^{ \pm}(A, *) \simeq C_{0}^{ \pm}\left(A^{\prime}, *^{\prime}\right)$.

When $\operatorname{deg}(A) \equiv 0(\bmod 4)$, we have the following relations in the Brauer group:

$$
2\left[C_{0}^{+}(A, *)\right]=[k] \quad 2\left[C_{0}^{-}(A, *)\right]=[k] \quad\left[C_{0}^{+}(A, *)\right]+\left[C_{0}^{-}(A, *)\right]=[A] .
$$

In this case, we need to consider also the noncommutative Chow motive of the involution variety $\operatorname{Iv}(A, *)$. Recall from [25, Example 3.11] that we have the following computation

$$
\begin{aligned}
U\left(\operatorname{perf}_{\mathrm{dg}}(\operatorname{Iv}(A, *))\right) \simeq & U(k)^{\oplus \frac{\operatorname{deg}(A)}{2}-1} \oplus U(A)^{\oplus \frac{\operatorname{deg}(A)}{2}-1} \\
& \oplus U\left(C_{0}^{+}(A, *)\right) \oplus U\left(C_{0}^{-}(A, *)\right)
\end{aligned}
$$


in the category $\operatorname{NChow}(k)$. If $[\operatorname{Iv}(A, *)]=\left[\operatorname{Iv}\left(A^{\prime}, *^{\prime}\right)\right]$ in the Grothendieck ring of varieties $K_{0} \operatorname{Var}(k)$, then $\mu_{\mathrm{JT}}([\operatorname{Iv}(A, *)])=\mu_{\mathrm{JT}}\left(\left[\operatorname{Iv}\left(A^{\prime}, *^{\prime}\right)\right]\right)$ in $R_{\mathrm{B}}(k)$. Thanks to Proposition 5.1(i), the latter equality holds if and only if we have an isomorphism $U\left(\operatorname{perf}_{\mathrm{dg}}(\operatorname{Iv}(A, *))\right) \simeq U\left(\operatorname{perf}_{\mathrm{dg}}\left(\operatorname{Iv}\left(A^{\prime}, *^{\prime}\right)\right)\right)$ in the category $\operatorname{NChow}(k)$. Note that the relations (12.3) imply, in particular, that $[A],\left[C_{0}^{+}(A, *)\right],\left[C_{0}^{-}(A, *)\right] \in{ }_{2} \operatorname{Br}(k)$. Therefore, making use of Proposition 4.7, we conclude that the noncommutative Chow motives $U\left(\operatorname{perf}_{\mathrm{dg}}(\operatorname{Iv}(A, *))\right)$ and $U\left(\operatorname{perf}_{\mathrm{dg}}\left(\operatorname{Iv}\left(A^{\prime}, *^{\prime}\right)\right)\right)$ are isomorphic if and only if the following two sets of Brauer classes

$$
\underbrace{\{[k]}_{\frac{\operatorname{deg}(A)}{2}-1}, \underbrace{[A]}_{\frac{\operatorname{deg}(A)}{2}-1},[\underbrace{\left[C_{0}^{+}(A, *)\right]}_{1},[\underbrace{\left[C_{0}^{-}(A, *)\right]}_{1}\} \underbrace{[[k]}_{\frac{\operatorname{deg}\left(A^{\prime}\right)}{2}-1}, \underbrace{\left[A^{\prime}\right]}_{\frac{\operatorname{deg}\left(A^{\prime}\right)}{2}-1},[\underbrace{\left[C_{0}^{+}\left(A^{\prime}, *^{\prime}\right)\right]}_{1},[\underbrace{\left[C_{0}^{-}\left(A^{\prime}, *^{\prime}\right)\right]}_{1}\}
$$

are the same up to permutation, where the numbers below the parenthesis denote the number of copies. Since $\operatorname{deg}(A) \geq 6$, there are at least 2 copies of $[k]$ and $[A]$. This fact, combined with the relations $(12.3)$, implies that $\left[C_{0}^{+}(A, *)\right]=\left[C_{0}^{+}\left(A^{\prime}, *^{\prime}\right)\right]$ and $\left[C_{0}^{-}(A, *)\right]=\left[C_{0}^{-}\left(A^{\prime}, *^{\prime}\right)\right]$ or that $\left[C_{0}^{+}(A, *)\right]=\left[C_{0}^{-}\left(A^{\prime}, *^{\prime}\right)\right]$ and $\left[C_{0}^{-}(A, *)\right]=$ $\left[C_{0}^{+}\left(A^{\prime}, *^{\prime}\right)\right]$. As above, using the equalities $\operatorname{deg}\left(C_{0}^{+}(A, *)\right)=\operatorname{deg}\left(C_{0}^{-}(A, *)\right)=$ $2^{\frac{\operatorname{deg}(A)}{2}-1}$ and $\operatorname{deg}(A)=\operatorname{deg}\left(A^{\prime}\right)$, we hence conclude that $C_{0}^{ \pm}(A, *) \simeq C_{0}^{ \pm}\left(A^{\prime}, *^{\prime}\right)$.

Item (iii). When $\operatorname{deg}(A)=6$, the assignment $(A, *) \mapsto C_{0}^{+}(A, *) \times C_{0}^{-}(A, *)$ gives rise to a one-to-one correspondence between isomorphism classes of central simple $k$-algebras of degree 6 with involution of orthogonal type and trivial discriminant and isomorphism classes of $k$-algebras of the form $Q \times Q^{\text {op }}$, where $Q$ is a quaternion algebra; consult [12, Cor. 15.32]. Note that the $k$-algebras $C_{0}^{+}(A, *) \times C_{0}^{-}(A, *)$ and $C_{0}^{+}\left(A^{\prime}, *^{\prime}\right) \times C_{0}^{-}\left(A^{\prime}, *^{\prime}\right)$ are isomorphic if and only if $C_{0}^{ \pm}(A, *) \simeq C_{0}^{ \pm}\left(A^{\prime}, *^{\prime}\right)$. Consequently, the proof follows from item (ii) and from the general fact that two central simple $k$-algebras with involution of orthogonal type $(A, *)$ and $\left(A^{\prime}, *^{\prime}\right)$ are isomorphic if and only if the involution varieties $\operatorname{Iv}(A, *)$ and $\operatorname{Iv}\left(A^{\prime}, *^{\prime}\right)$ are isomorphic.

Item (iv). When $I^{3}(k)=0$, we have the following classification theorem: if $\operatorname{deg}(A)=\operatorname{deg}\left(A^{\prime}\right)$ and $C_{0}^{ \pm}(A, *) \simeq C_{0}^{ \pm}\left(A^{\prime}, *^{\prime}\right)$, then the central simple algebras with involution of orthogonal type $(A, *)$ and $\left(A^{\prime}, *^{\prime}\right)$ are isomorphic; consult [20, Thm. A]. Consequently, the proof follows from the combination of items (i)-(ii) with the general fact that two central simple $k$-algebras with involution of orthogonal type $(A, *)$ and $\left(A^{\prime}, *^{\prime}\right)$ are isomorphic if and only if the involution varieties $\operatorname{Iv}(A, *)$ and $\operatorname{Iv}\left(A^{\prime}, *^{\prime}\right)$ are isomorphic.

Acknowledgements I am grateful to Michael Artin for enlightning discussions about Severi-Brauer varieties, to Marcello Bernardara for a stimulating discussion about the Amitsur's conjecture, to Asher Auel for the references $[8,20]$, and to the anonymous referees for their comments. I am also very grateful to the Institut des Hautes Études Scientifiques (IHÉS) and to the Max-Planck-Institut für Mathematik (MPIM) for their hospitality, where this work was finalized.

Data Availability Data sharing not applicable to this article as no datasets were generated or analysed during the current study. 


\section{Declarations}

Conflict of interest The author states that there is no conflict of interest.

Open Access This article is licensed under a Creative Commons Attribution 4.0 International License, which permits use, sharing, adaptation, distribution and reproduction in any medium or format, as long as you give appropriate credit to the original author(s) and the source, provide a link to the Creative Commons licence, and indicate if changes were made. The images or other third party material in this article are included in the article's Creative Commons licence, unless indicated otherwise in a credit line to the material. If material is not included in the article's Creative Commons licence and your intended use is not permitted by statutory regulation or exceeds the permitted use, you will need to obtain permission directly from the copyright holder. To view a copy of this licence, visit http://creativecommons.org/licenses/by/4.0/.

\section{References}

1. Albert, A.: Tensor products of quaternion algebras. Proc. AMS 35, 65-66 (1972)

2. Amitsur, S.: Generic splitting fields of central simple algebras. Ann. Math. 62, 8-43 (1955)

3. Bittner, F.: The universal Euler characteristic for varieties of characteristic zero. Compos. Math. 140(4), 1011-1032 (2004)

4. Bondal, A., Kapranov, M.: Framed triangulated categories. Mat. Sb. 181(5), 669-683 (1990)

5. Bondal, A., Orlov, D.: Derived categories of coherent sheaves. Proceedings of the international congress of mathematicians, Vol. II, pp. 47-56. Higher Ed. Press, Beijing (2002)

6. Bondal, A., Orlov, D.: Semiorthogonal decomposition for algebraic varieties. Available at arXiv:alg-geom/9506012 (2021)

7. Colmez, P., Serre, J.-P.: Correspondance Grothendieck-Serre. Doc. Math. 2 (2001)

8. Elman, R., Lam, T.-Y.: Classification theorems for quadratic forms over fields. Comment. Math. Helv. 49, 373-381 (1974)

9. Gille, P., Szamuely, T.: Central simple algebras and Galois cohomology, vol. 101. Cambridge Studies in Advanced Mathematics. Cambridge University Press, Cambridge (2006)

10. Hogadi, A.: Products of Brauer-Severi surfaces. Proc. AMS 137(1), 45-50 (2009)

11. Keller, B.: On differential graded categories. International congress of mathematicians (Madrid), Vol. II, pp. 151-190. The European Mathematical Society, Zürich (2006)

12. Knus, M.-A., Merkurjev, A., Rost, M., Tignol, J.-P.: The book of involutions. With a preface in French by J. Tits, vol. 44. AMS Colloquium Publications, Providence (1998)

13. Kollár, J.: Conics in the Grothendieck ring. Adv. Math. 198(1), 27-35 (2005)

14. Kollár, J., Smith, K., Corti, A.: Rational and nearly rational varieties. Cambridge Studies in Advanced Mathematics, vol. 92. Cambridge University Press, Cambridge (2004)

15. Kontsevich, M.: Noncommutative motives. Talk at the IAS on the occasion of the $61^{\text {st }}$ birthday of Pierre Deligne. Available at http://video.ias.edu/Geometry-and-Arithmetic (2005)

16. Kontsevich, M.: Notes on motives in finite characteristic. Algebra, arithmetic, and geometry: in honor of Yu I Manin. Vol. II, pp. 213-247, Progr. Math., 270, Birkhäuser, Boston (2009)

17. Kontsevich, M.: Mixed noncommutative motives. Talk at the workshop on homological mirror symmetry, Miami. Available at http://www-math.mit.edu/auroux/frg/miami10-notes (2010)

18. Lam, T.-Y.: Introduction to quadratic forms over fields. Graduate Studies in Mathematics, vol. 67. American Mathematical Society, Providence (2005)

19. Larsen, M., Lunts, V.: Motivic measures and stable birational geometry. Mosc. Math. J. 3(1), 85-95 (2003)

20. Lewis, D., Tignol, J.-P.: Classification theorems for central simple algebras with involution (with an appendix by R. Parimala). Manuscr. Math. 100, 259-276 (1999)

21. Orlov, D.: Projective bundles, monoidal transformations, and derived categories of coherent sheaves. Izv. Ross. Akad. Nauk. Ser. Mat. 56(4), 852-862 (1992)

22. Panin, I.: On the algebraic $K$-theory of twisted flag varieties. K-Theory 8(6), 541-585 (1994)

23. Roquette, P.: Isomorphisms of generic splitting fields of simple algebras. J. Reine Angew. Math. 214(215), 207-226 (1964) 
24. Steinberg, R.: On a theorem of Pittie. Topology 14, 173-177 (1975)

25. Tabuada, G.: Additive invariants of toric and twisted projective homogeneous varieties via noncommutative motives. J. Algebr. 417, 15-38 (2014)

26. Tabuada, G.: Noncommutative Motives. With a preface by Yuri I. Manin. University Lecture Series., vol. 63. American Mathematical Society, Providence (2015)

27. Tabuada, G.: Noncommutative motives in positive characteristic and their applications. Adv. Math. 349, 648-681 (2019)

28. Tabuada, G., Van den Bergh, M.: Noncommutative motives of separable algebras. Adv. Math. 303, 1122-1161 (2016)

29. Tabuada, G., Van den Bergh, M.: The Gysin triangle via localization and $\mathbb{A}^{1}$-homotopy invariance. Trans. AMS 370(1), 421-446 (2018)

30. Tits, J.: Représentations linéaires irréductibles d'un groupe réductif sur un corps quelconque. J. Reine Angew. Math. 247, 196-220 (1971)

31. Tregub, S.: Birational equivalence of Brauer-Severi manifolds. Uspekhi Mat. Nauk 46(6), 217-218 (1991); Transl. Russ. Math. Surv. 46 (6), 229 (1991)

Publisher's Note Springer Nature remains neutral with regard to jurisdictional claims in published maps and institutional affiliations. 